\title{
Shame Sanctions - Ehrenstrafen im Lichte der Straftheorie
}

Von Wiss. Ass. Dr. Michael Kubiciel, Regensburg

\section{Einführung}

Eine Frau, der man Ehebruch vorwarf, wird an einem gut sichtbaren Ort der Menge vorgeführt und sodann auf einem Schandwagen durch die Stadt gefahren. Ein anderer Ort, ein vergleichbares Vorgehen: Kundennamen von Prostituierten werden in Zeitungen, an Anschlagbrettern oder im kommunalen Fernsehen veröffentlicht. Das erste Beispiel wird uns aus dem antiken Griechenland überliefert ${ }^{1}$, das zweite Beispiel ist Praxis in einigen Bundesstaaten der USA am Ausgang des 20. Jahrhunderts ${ }^{2}$. Zwischen den Beispielen liegt nicht nur ein Zeitraum von zweitausend Jahren. Sie trennt der Epochenwandel der Aufklärung, eine Zeitenwende, welche auch das strafrechtliche Sanktionensystem nicht unberührt ließ ${ }^{3}$.

Die Renaissance der Ehrenstrafe macht den europäischen Betrachter ratlos: Ist die amerikanische Rechtspolitik in einer anachronistischen Wendung auf eine Sanktionsart einer vergangenen Zeit gestoßen? Oder sind die shame sanctions nur ein weiteres Zeichen dafür, dass die amerikanische Gesellschaft nicht etwa einem kulturellen Standard Europas hinterher hinkt, sondern einer möglichen Entwicklung diesseits des Atlantiks voraus eilt? Ließen sich shame sanctions nämlich als ein Mittel zur Manifestation gemeinsamer Werte, als Antwort auf die Zentrifugalkräfte eines seit jeher von kultureller Offenheit, Liberalität und Pluralismus geprägten Landes verstehen ${ }^{4}$, könnten derartige Phänomene bald auch in unserer Mitte sichtbar werden. Denn seitdem immer deutlicher wird, dass auch uns weite Freiheitsräume nicht kostenlos

1 Plutarch, Moralische Schriften, Fragen über griechische Gebräuche, S. 2, zitiert nach v. Hentig, Die Strafe, Band I, Frühformen und kulturgeschichtliche Zusammenhänge, 1954, S. 400.

2 Kahan, University of Chicago Law Review Vol. 63 (1996), S. 591, 632; Whitman, The Yale Law Journal, Vol. 107 (1998), S. 1055, 1056.

3 Siehe dazu etwa Seelmann, ZStW 101 (1989), S. 335 ff., 347 ff.; Nagler, Die Strafe, 1918, S. $289 \mathrm{ff}$; Whitman (Anm. 2), S. $1069 \mathrm{ff}$.

4 Vgl. dazu Tonry, Thinking about Crime, Oxford, 2004, S. 157, der die auch in den shame sanctions zum Ausdruck kommenden Tendenzen einer „increased attention to the norm-reinforcement, moral-educative, and expressive effects of punishment" $\mathrm{zu}$ schreibt. 
erschlossen worden sind, sondern mit dem Verlust an gemeinsamen Werten erkauft wurden ${ }^{5}$, laboriert auch das Strafrecht hierzulande in einem zunehmend wertkonsensfreien Raum. Ob das Strafrecht in einer pluralistischen Gesellschaft überhaupt noch als sittenbildende $\mathrm{Kraft}^{6}$, moderner: als Mittel zur Einübung in Normanerkennung ${ }^{7}$, wirken kann und soll, ist Teil des Streits um den Sinn der Strafe ${ }^{8}$.

Ungeachtet dieses Streits dürften Ehrenstrafen hierzulande auf breite Ablehnung in der Strafrechtswissenschaft stoßen, passen sie doch nicht zu der seit etwa 200 Jahren zu beobachtenden Distanzierung der Strafe von der Person des Täters. Diese Entwicklung ist zweifellos auch Ausdruck einer bürgerlichen Ästhetik, welche sich dem Strafen, wenn überhaupt, nur mit spitzen Fingern nähert. „Es ist hässlich straffällig zu sein - und wenig ruhmvoll, strafen zu müssen “, bemerkt Foucault treffend ${ }^{9}$. Tatsächlich ist uns die Konfrontation mit der Strafe fremd geworden. Gefängnismauern haben uns den Blick auf den Akt der Bestrafung verstellt, die Geldstrafe kommt sogar völlig ohne sichtbare Symbolik aus. Wenn es aber nur ein ästhetisches Unbehagen ist, welches den Blick des Bürgers von dem Delinquenten nimmt und zur „Heimlichkeit“ 10 des Strafens führt, können tatsächlich mit dem Siegeszug des „humor of the bedroom and the bathroom"11 auch hierzulande Rufe nach einer Öffentlichkeit des Strafens ertönen ${ }^{12}$. Das Auftauchen der Ehrenstrafen hat jedenfalls die Überzeugung widerlegt, die gesellschaftliche Entwicklung führe zu einer Überwindung ${ }^{13}$, wenigstens aber zu einer „Zivi-

5 Erst die wirtschaftliche und gesellschaftliche Entwicklung der letzten Jahre habe uns, wie Kersting, Cicero 10/2004, S. 98, 100, meint, vor Augen geführt, dass wir auch diesseits des Atlantiks einem Modernitätspensum ausgesetzt seien, welches dem Individuum vor einem „offenen, leeren Himmel“ schwer falle zu erfüllen.

6 Welzel, Das deutsche Strafrecht, 11. Aufl. 1969, S. 4; dagegen Mir Puig, ZStW 102 (1999), S. 914, 923.

7 Jakobs, Strafrecht Allgemeiner Teil, 2. Aufl. 1993, 1. Abschnitt Rdn. 15.

8 Umfassend dazu zuletzt Pawlik, Person, Subjekt, Bürger, 2004.

9 Foucault, Überwachen und Strafen, 1996, S. 17; siehe auch Whitman (Anm. 2), S. 1055: „It was best to keep the fact of crime hidden, for knowledge of crime tended to corrupt the knower".

10 Nagler (Anm. 3), S. 111: „Der Vollzug sucht unbeschadet seines Ernstes die Heimlichkeit und entbehrt alles der äußeren Eindrücke berechneten Gepränges“.

11 Treffend Whitman (Anm. 2), S. 1079.

12 Siehe dazu den Essay von Pawlik, FAZ vom 17.11.2004, S. 35.

13 Siehe dazu schon Radbruch, Rechtsphilosophie, 7. Aufl. 1970, S. 269, der prophezeite, dass die Entwicklung über das Strafrecht hinwegschreiten werde. 
lisierung“14 der Strafe ${ }^{15}$. Im Zuge der kulturellen Evolution einer Gesellschaft ist eine zivilisierte Strafe allenfalls dann von Dauer, wenn sich "gute“ normative Gründe nennen lassen, weshalb bestimmte Strafformen mit unserer Gesellschafts- und Rechtsordnung unverträglich sind. Will man dazu nicht voreilig und pauschal auf die - im Bereich des Strafens keineswegs unverrückbare ${ }^{16}$ - Menschenwürde verweisen, muss sich das Strafrechtssystem selbst Rechenschaft darüber ablegen, ob es mit der Einführung einer Sanktionsart wie der Ehrenstrafe seine Funktionen verfehlte oder gar die Dignität verlöre.

Die Betrachtung der shame sanctions dient mithin nicht allein dem rechtsvergleichenden Erkenntnisgewinn über die Diskussion in den USA (II). Die Thematik eignet sich in besonderem Maße zur Beantwortung der Frage, ob das deutsche Strafrechtssystem bestimmte Strafarten ausschließt oder ob es derart vom Entwicklungsstand der Gesellschaft abhängt ${ }^{17}$, dass es sich der Mittel bedienen darf, welche zur Erfüllung der „Schutzaufgabe des Staates unter den Lebensbedingungen der pluralistischen Gesellschaft “ 18 benötigt werden. Die Antwort hierauf könnte in einem Verständnis der Strafe als wertbezogene und wertgebundene Institution liegen (III).

\section{Die shame sanctions in der amerikanischen Diskussion}

1. Die shame sanctions im Lichte der amerikanischen Strafrechtspolitik und Straftheorien

a) Formen und Wesenszüge der shame sanctions

Ehrenstrafen werden in den USA vor allem gegenüber Ersttätern und als Antwort auf Bagatell- und Massendelikte verhängt. So folgt nicht selten auf eine Trunkenheitsfahrt die Strafe, sich mit einem Schild „I am a drunken driver“ vor das Gerichtsgebäude zu stellen oder einen entsprechenden Auf-

14 Vgl. Kunz, Kriminologie, 2003, \41 Rdn. 3; 442 Rdn. 25: „Die langfristige historische Entwicklung geht auf eine Milderung des Strafübels“.

15 Vgl. auch Jung, Sanktionensysteme und Menschenrechte, 1991, S. 41, der zutreffend bemerkt, dass die Ausbildung von Strafsensibilität in der Gesellschaft nicht heißen müsse, dass der Zugriff auf die Person zwangsläufig stetig und berechenbar abnehmen müsse.

16 Dazu Herdegen, in: Maunz/Dürig, Grundgesetz Kommentar, 2003, Art. 1 Abs. 1 Rdn. 43 ff., unter Verweis auf „abwägungsgeleitete“ Urteile des BVerfG.

17 Jakobs (Anm. 7), 1. Abschnitt Rdn. 1.

18 Jescheck/Weigend, Lehrbuch des Strafrechts, Allgemeiner Teil, 5. Aufl. 1996, S. 2. 
kleber am Auto anzubringen ${ }^{19}$. Auch anderen Formen verbreiteter Delinquenz, namentlich dem Ladendiebstahl, begegnet man mit derartigen Sanktionen ${ }^{20}$. Den Einsatz in gerade diesem Deliktsfeld erklärt Whitman mit der Zielrichtung, Erst- und Gelegenheitstätern mit dem öffentlichen Strafvollzug vor Augen zu führen, dass die Begehung weiterer Straftaten einen gesellschaftlichen Statutswechsel mit sich bringen könne, der ihnen dauerhaft das Gefühl vermitteln werde, welches sie während des Vollzugs der Ehrenstrafe empfänden ${ }^{21}$. Ein anderes Anwendungsfeld der shame sanctions sind Straftaten, die Verhaltensweisen pönalisieren, welche als anstößig erachtet werden („moral offences“) ${ }^{22}$. Als Paradebeispiel für die beabsichtigte Wirkungsweise kann insoweit die Veröffentlichung der Kundennamen von Prostituierten gelten ${ }^{23}$ : Offenkundig ist die öffentliche Bekanntmachung als geeignetes Strafübel gegen Verhaltensweisen (wieder-)entdeckt worden, die auf Verheimlichung angelegt sind und gegen die demzufolge eine Kombination staatlicher und gesellschaftlicher, formeller und informeller Sanktionen als besonders wirksame Form der Sozialkontrolle genutzt werden kann ${ }^{24}$. Unter die Kategorie der „moral offences“ lässt sich auch das Vorgehen gegen „Wall-Street-Broker“ rubrizieren, die zur öffentlichen Reinigung des von ihnen durch Urin verschmutzten Gehwegs angehalten worden sind ${ }^{25}$. Gerade an diesem Beispiel wird ein besonderer Wesenszug der shame sanctions deutlich: Ein System, welches die Oberschicht von ehrverletzenden Strafen nicht mehr verschont ${ }^{26}$, sondern shame sanctions gegen Personen, die auf ihre Reputation geschäftlich angewiesen sind, verstärkt verhängt ${ }^{27}$, zielt auf den Bereich, in welchem die Personen besonders schmerzhaft getroffen wer-

Siehe Kahan (Anm. 2), S. 632; Whitman (Anm. 2), S. 1056.

Kaban (Anm. 2), S. 633.

Whitman (Anm. 2), S. 1067f., betrachtet den Vollzug der shame sanctions in diesen Fällen daher als „liminal rites, rites that warn first and minor offenders that they are flirting with a deep, and deeply undesirable, status change“.

Siehe Whitman (Anm. 2), S. 1064.

Siehe die Nachweise in Anm. 2.

Foucault (Anm. 9), S. 41, würde hierin einen weiteren Beleg für seine These finden, nach welcher sich der Zugriff des Staates vom Körper weg hin zur Innerlichkeit, zur „Seele“, des Täters bewege, die ihrerseits „den aktuellen Bezugspunkt einer bestimmten Technologie der Macht über den Körper“ darstelle.

Kaban (Anm. 2), S. 633.

v. Hentig (Anm. 1), S. 412 f.; siehe auch Schwerhoff, in: Blaubert/Schwerhoff (Hrsg.), Mit den Waffen der Justiz, 1998, S. 158, 174ff., der aus dem deutschen Kulturkreis eine Vielzahl von Belegen nennt, dass Menschen mit Geld und Ansehen in der Lage waren, sich durch Geldzahlungen von Ehrenstrafen freizukaufen.

Whitman (Anm. 2), S. 1066 f. 
den können: die gesellschaftliche Anerkennung. Schließlich fügt auch die unter der Bezeichnung „Megan’s Law“ 28 bekannt gewordene Maßnahme, die Häuser freigelassener Sexualtäter mit Schildern zu kennzeichnen, ein den übrigen shame sanctions vergleichbares Übel $\mathrm{zu}^{29}$. Doch wird dieser Kennzeichnungspflicht zuvorderst ein prospektiv-gefahrenabwehrender Charakter zugeschrieben ${ }^{30}$, da nicht mehr die vergangene Tat gesühnt, sondern von dieser auf die Gefährlichkeit des Täters geschlossen wird. Im zweispurigen deutschen System ließe sich das Megan's Law folglich als eine Maßregel der Sicherung bezeichnen. Das amerikanische Pendant teilt freilich die Eigenschaften deutscher Maßregeln, vom Täter und der Öffentlichkeit als Strafe empfunden zu werden ${ }^{31}$, und zudem ein Übel zuzufügen, welches das der Strafe weit überschreiten kann ${ }^{32}$.

Angesichts dieser Vielgestaltigkeit der shame sanctions fragt sich, ob die Kreativität der Sanktionierung mit einem strafrechtstheoretischen Konzept in Einklang zu bringen ist, welches als Grundlage für eine kritische Auseinandersetzung dienen kann. Dies wird selbst in den USA bestritten. Michael Tonry hat der amerikanischen Strafrechtspolitik jüngst vorgehalten, sich im Wald guter Absichten, öffentlicher Ängste und politischer Zynismen verlaufen und ein Strafsystem geschaffen zu haben, auf welches sich niemand sehenden Auges verständigt hätte ${ }^{33}$. Tatsächlich sind shame sanctions $\mathrm{Be}-$ standteil eines umfangreichen Strafarsenals, das auch in den USA vor Jahren

Kaban (Anm. 2), S. 632 f.; Teichman (Anm. 29), S. 28.

1 Siehe zur Ähnlichkeit von Strafe und Maßregel Jescheck/Weigend (Anm. 18), S. 84; Roxin, Strafrecht Allgemeiner Teil 1, 3. Aufl. 1997, \3 Rdn. 58: „Es ergibt sich also, daß der Zweck von Strafe und Maßregel sich im wesentlichen nicht unterscheidet. [...] Strafe und Maßregel weichen also nicht im Ziel, sondern in der Begrenzung von einander $a b^{\prime \prime}$.

32 Besonders deutlich wird dies, wenn man sich vergegenwärtigt, dass durch die Kennzeichnung der Häuser gleichsam deren Mauern aufgesprengt werden, in deren Befriedung sich der Einzelne zurückzieht, um sich von der Öffentlichkeit absondern zu können (dazu Canetti, Masse und Macht, 2003, S. 19). Der Zugriff wird umfassend, weil dem (freigelassenen) Delinquenten der Rückzugsraum verbaut wird, in welchem er grundsätzlich das Recht haben soll, in Ruhe gelassen zu werden, weil er die Sozialsphäre des rechtlich regulierungsbedürftigen Raums nicht betritt, dazu BVerfGE 109, $279,309$.

33 Tonry (Anm. 4), S. 3: „American policy makers got lost in a forest of good intentions, public anxieties, and politicial cynicism. They created a punishment system that no one would knowingly have chosen, but that we do not know how to change“. 
noch „undenkbar“ gewesen wäre ${ }^{34}$ und in Deutschland (noch) undenkbar ist. Wenn aber das amerikanische Sanktionensystem allein Ausdruck einer ausschließlich am Wähler orientierten, simplifizierenden Strafrechtspolitik ist, wie Tonry meint ${ }^{35}$, könnte sich die deutsche Strafrechtswissenschaft in der Gewissheit zurücklehnen, dass sich derartige Bestrebungen hierzulande im Dickicht strafrechtstheoretischer Erkenntnisse verfingen. Wenn hier dennoch der Versuch unternommen wird, die shame sanctions mit jüngeren Tendenzen in der amerikanischen Kriminalpolitik und Straftheorie abzugleichen, sollen nicht nur die Wesenszüge der Ehrenstrafen herausgearbeitet, sondern soll vor allem Tonrys These auf ihre Tragfähigkeit untersucht werden.

\section{b) Zuordnung der shame sanctions zu anglo-amerikanischen Straftheorien}

Bis in die 1970er Jahre dominierte eine rehabilitationsorientierte spezialpräventive Straftheorie die amerikanische Rechtspolitik und Strafpraxis ${ }^{36}$. Diese erblickte den Sinn der Strafe nicht in einem Ausgleich für verwirkte Schuld, sondern als Mittel zur Besserung des Straftäters und damit zur Verhinderung künftiger Straftaten. Nachdem sich der Eindruck festgesetzt hatte, dass Rehabilitationsmaßnahmen kaum Wirkung zeitigten („nothing works“37) und sich die an sog. Parole Boards delegierte Entscheidung über die Dauer der Freiheitsstrafe wachsender Kritik ausgesetzt sah ${ }^{38}$, vollzog die amerikanische Kriminalpolitik eine Wendung hin zu einem retributiven „just-desert“-Modell ${ }^{39}$. Statt der Besserung des Delinquenten sollte nunmehr der Tadel in den Vordergrund treten und dieser die Vorwerfbarkeit der Tat widerspiegeln ${ }^{40}$. Indem der Tadel nicht mehr als ein - die Reintegration

Siehe Tonry (Anm. 4), S. $141 \mathrm{ff}$. Neben der Schamzufügung als Strafe wird über das „real offense sentencing" berichtet, welches einem Richter die Einbeziehung von „Straftaten“ in die Strafzumessung erlaubt, derentwegen der Angeklagte nicht angeklagt oder freigesprochen worden ist. Der Richter ist bei der Einbeziehung solcher Punkte von der grundsätzlichen Verpflichtung, es dürfe kein Zweifel an der Schuld des Angeklagten bestehen, befreit, sondern muss die Begehung der Tat lediglich für wahrscheinlich halten. Tonry (Anm. 4), S. 200.

36 Dazu Weigend, Festschrift der Rechtswissenschaftlichen Fakultät zur 600-Jahr-Feier der Universität zu Köln, 1988, S. 579, 586 ff.

37 Siehe zu diesem Befund Duff/Garland, in: dies., A Reader on Punishment, Oxford, 1995, S. 10.

38 Banks, Criminal Justice and Ethics, Flagstaff, 2004, S. 113.

39 Dazu Duff/Garland, in: dies. (Anm. 37), S. 8 ff.; Banks (Anm. 38), S. 104, 113.

40 v. Hirsch, in: Walker (Hrsg.), Why Punish?, Oxford, 1991, S. 78. 
behinderndes - Stigma, sondern als Voraussetzung für die Anerkennung der Verfehlung und als Mittel zur Verhinderung zukünftiger Verfehlung erachtet wurde ${ }^{41}$, avancierte er vom schamhaft verleugneten Stiefkind der Straftheorie zu ihrem zentralen Bestandteil. In den 1980er Jahren hat sich das ,just-desert"-Modell durchgesetzt und kann als die einflussreichste Straftheorie in den USA bezeichnet werden ${ }^{42}$. Im zeitlichen Zusammenhang mit der Etablierung dieser vergeltenden Strafzwecktheorie werden auch shame sanctions in einigen Bundesstaaten der USA virulent ${ }^{43}$.

Die zeitliche Koinzidenz legt die Vermutung nahe, dass die shame sanctions nicht zufällig auftauchten, sondern von dieser neuen Strömung der Straftheorie getragen wurden. Tatsächlich findet der Tadel als integrales Merkmal der ,just-desert“-Theorie gerade in den Ehrenstrafen einen besonders greifbaren Ausdruck. Aus diesem Grund hat namentlich Kaban dem „shaming“ die Fähigkeit zuerkannt, in vergleichbarem Maße wie Gefängnisstrafen die „moralische Verurteilung“ zu artikulieren und daher auch von der Allgemeinheit als alternative Strafform akzeptiert zu werden ${ }^{44}$. Auch mit der zweiten Komponente des „just-desert“-Ansatzes, der Schuldangemessenheit, lassen sich shame sanctions jedenfalls dann vereinbaren, wenn man sie als Ersatz für Freiheitsstrafen anwendet ${ }^{45}$. Schwierigkeiten dürfte den Befürwortern der shame sanctions freilich das Postulat des „just desert“Modells bereiten, den Delinquenten als „moral agent“ zu behandeln ${ }^{46}$. Immerhin ließe sich vorbringen, der Verurteilte werde beim Tragen eines Zeichens zum Objekt eines „infantilisierenden “ 47 Strafregimes degradiert, das dem Täter die Einsicht in die Fehlsamkeit seines Tuns eben nicht mittels der Einwirkung auf seine Überzeugungen vermitteln wolle.

Demgegenüber betont $v$. Hirsch, dass eine Änderung der inneren Überzeugung des Straftäters zwar wünschenswert, nicht aber das Ziel der Strafe sein könne, erfordere dies doch nicht nur einen Einblick in die Persönlichkeit und einen Ausblick auf seine Änderungsfähigkeit, sondern verkehrte die Funktion des strafenden Staats: „The condemnor's role is not of the mentor

41 Siehe dazu Banks (Anm. 38), S. 113 f.

42 Banks (Anm. 38), S. 113.

43 Kahan (Anm. 2), S. 631.

44 Kahan (Anm. 2), S. 635 f.; kritisch dazu Tonry (Anm. 33), S.156ff., der meint, Ehrenstrafen ließen sich aus dem etwa von Duff vertretenen expressiven Strafverständnis nicht ableiten (dazu unten Anm. 52).

45 Kahan (Anm. 2), S. $631 \mathrm{ff}$.

46 v. Hirsch, in: Duff/Garland (Anm. 37), S. 115, 121.

47 Siehe Duff/Garland, in: dies. (Anm. 37), S. 27. 
or priest“ ${ }^{48}$. Die im Tadel zum Ausdruck kommende Wertsetzung soll dem Täter also nicht anerzogen werden. Der Delinquent kann vielmehr die Vollstreckung der shame sanction wie einen Freiheitsentzug auch bloß passiv hinnehmen. Mehr noch: Indem ihm die Alternativen eröffnet sind, die Strafe entweder zur inneren Umkehr zu nutzen oder aber nur passiv zu erdulden, kann er seine Eigenschaft als „moral agent“ bestätigt sehen. Erst dann, wenn die Sanktionsdrohung derart schwerwiegend sei, dass die abschreckende Wirkung jede „normative Botschaft" verdecke, behandele man den Menschen nicht mehr als einen verantwortlich Handelnden ${ }^{49}$. Dass die Androhung der shame sanctions den Wertgehalt der Norm verdeckt, kann aber, wie noch zu zeigen sein wird, nicht behauptet werden.

Höher sind freilich die Anforderungen an die Strafe und ihre Vollstreckung, wenn man, wie Duff, in ihr eine Form weltlicher Buße erblickt, die dem Täter eine „self-reform" abverlange und ermögliche ${ }^{50}$. Knüpft man die Erwartung eines moralischen Urteils des Täters über sich und seine Tat an eine aktive Rolle des Delinquenten im Kommunikationsprozess ${ }^{51}$, dann scheint der den Ehrenstrafen zugrunde liegende Kommunikationsprozess wenig geeignet, dem Täter zu einer „self-reform“ zu verhelfen. Die Ehrenstrafe und ihr Tadel vermitteln sich ihm nämlich mehr durch die Auslösung einseitiger Empfindungen bei den Betrachtern als in einer wechselseitigen Kommunikation mit dem strafenden Staat ${ }^{52}$. Dies legt freilich die Vermutung nahe, die shame sanctions verfolgten erst gar nicht das Ziel, an das Normbewusstsein des Delinquenten appellieren zu wollen ${ }^{53}$. Tatsächlich wird die besondere Kommunikationsrichtung der Ehrenstrafen sichtbar,

v. Hirsch, in: Duff/Garland (Anm. 37), S. 115, 120.

v. Hirsch, in: Tonry (Hrsg.), The Handbook of Crime and Punishment, New York/Oxford, 1998, S. 659, 667; v. Hirsch/Hörnle, GA 1995, 261, 279.

Siehe Duff, Punishment, Communication, and Community, 2001, S. 106: „a species of secular penence“. Wenn Banks (Anm. 38), S. 114, freilich meint, dass sämtliche retributiven Modelle dem Täter ein „moralisches Urteil“ abverlangten, verkennt sie, dass v. Hirsch, in: Duff/Garland (Anm. 37), S. 120, diesen Anspruch ausdrücklich zurückweist.

1 Siehe dazu Banks (Anm. 38), S. 111 m. w. N.

2 Vgl. zur Notwendigkeit einer wechselseitigen Kommunikation Duff (Anm. 50), S. 79: „Although some theorists talk of ,expressive' purpose of punishment [...] we should rather talk of its communicative purpose: for communication involves, as expression need not, a reciprocal and rational engagement".

Freilich teilen die Ehrenstrafen den Effekt, nicht als wechselseitiger Kommunikationsprozess verstanden zu werden, mit „konventionellen“ Strafen, dürfte doch auch das Gefängnis seinen Insassen kaum als geeigneter Ort kommunikativer Verständigung über die Verfehlung und einer daraus resultierenden Läuterung erscheinen. 
wenn man der Sprache der Strafe einen zweiten Adressaten zuweist: die Allgemeinheit. Der in der Strafe symbolisierte Tadel soll dieser dann, wie v. Hirsch formuliert, „Gründe liefern“, von der Begehung entsprechender Taten abzusehen ${ }^{54}$. Als einen derartigen Grund erkennen potentielle Täter die in der Sanktionsdrohung angekündigte Rechtsgutsentziehung. Die Strafandrohung soll mithin abschrecken ${ }^{55}$. Freilich verträgt sich der Rückgriff auf derartige Klugheitsgründe eines nutzenabwägenden Individuums nur schwer mit der Legitimationsgrundlage der „just-desert“-Theorie, Normadressaten sollten als vernünftige und nicht als triebgesteuerte Menschen angesehen werden ${ }^{56}$. Dies mag ein Grund sein, weshalb v. Hirsch die negativabschreckende Komponente durch eine positive ergänzt: „Through the censure expressed by such sanctions, the law registers disapprobation of the behaviour. Citizens are thus provided with moral and not just prudential reasons for desistence “ 57 . Ohne den innersystematischen Zusammenhang zwischen der vergeltenden und präventiven Komponente auszuarbeiten, wird dem Strafvollzug nicht nur die Vergeltung der Schuld, sondern werden ihm zugleich die Aufgaben der positiven und - gewissermaßen: „hilfsweise“ negativen Generalprävention zugewiesen. Zwar sind shame sanctions im Vergleich zu Freiheitsstrafen weniger abschreckend. Dem Anspruch positiver Generalprävention werden sie aber in besonderem Maße gerecht: Im Gegensatz zu den außerhalb des Blicks der Öffentlichkeit vollzogenen Freiheits- und Geldstrafen liefern sie dem Betrachter in eindrücklicher Weise eine werthaltige Botschaft und damit die geforderten „moral reasons“ für normkonformes Verhalten: Das Schild „I write bad checks“ lesen die Betrachter nicht nur als „Don't write bad checks!“. Sie sollen den Satz auch im Sinne von „Writing bad checks is immoral!“ verstehen.

Tonrys These, das amerikanische Sanktionensystem orientiere sich mehr an vermuteten Wählerinteressen als dass es Ausdruck strafrechtstheoretischer Erwägungen sei, sieht sich von den shame sanctions mithin nicht bestätigt: Sie lassen sich nicht nur in die jüngere kriminalpolitische Entwick-

v. Hirsch, in: Duff/Garland (Anm. 37), S. 115, 120: „The criminal law gives the censure it expresses yet another role: that of addressing third parties, and providing them with reasons for desistence."; siehe auch Banks (Anm. 38), S. 110; zur expressiven Funktion der Strafe auch Feinberg, in: Duff/Garland (Anm. 37), S. 71, 74. drohung einen Klugheitsgrund, einer strafbaren Neigung zu widerstehen, wenn die moralische Intuition versage (a.a.O., S. 123). 
lung in den USA einordnen, sondern scheinen auch sämtliche strafrechtstheoretischen Sinnzuschreibungen der Strafe zu erfüllen: Ehrenstrafen sind ein probates Mittel eines retributiven Strafmodells und sie wirken, wenngleich weniger stark als die Freiheitsstrafe, abschreckend. Teilt man die Auffassung Whitmans von den shame sanctions als „liminal rite“ für Erst- und Gelegenheitstäter ${ }^{58}$, lässt sich grundsätzlich auch ein Rehabilitationseffekt nicht leugnen. Vor allem aber sind sie ein besonders geeignetes Mittel der positiven Generalprävention ${ }^{59}$. Dass sie diese auf positive Generalprävention zielende Absicht mit den mittelalterlichen und frühneuzeitlichen Ehrenstrafen in Deutschland teilen ${ }^{60}$, legt die Vermutung nahe, dass sie ihre Renaissance gerade der Eignung verdanken, an Werte und Normen zu erinnern, die ungeachtet ihrer häufigen Verletzung für unverfügbar erachtet werden ${ }^{61}$. Unabhängig aber von der Frage, welcher Straftheorie die Ehrenstrafen im besonderen Maße gerecht werden, vermittelt der bisherige Befund unserer Untersuchung eine beunruhigende Botschaft: Wenn sich die shame sanctions strafrechtstheoretisch erklären und rechtfertigen lassen, kann sich die intuitive Ablehnung solcher Sanktionen nicht auf einen evidenten normativen Grund stützen. Die Suche nach solchen Gründen muss also fortgesetzt werden. Im Folgenden soll zunächst die amerikanische Kritik zu Wort kommen.

2. Die shame sanctions in der Kritik der amerikanischen Strafrechtswissenschaft

Lässt man den „funktionalen“ Einwand beiseite, shame sanctions versagten in einer modernen, anonymen Gesellschaft ${ }^{62}$, dann sieht sich diese Strafform in den USA mit zwei normativen Kritikpunkten konfrontiert: Zum einen wird behauptet, shame sanctions griffen in unzulässiger Weise auf die Innerlichkeit der Normadressaten zu (dazu a). Zum zweiten werden die von Ehrenstrafen ausgehenden Gefahren für die Gemeinschaft und die Friedensordnung hervorgehoben (dazu b).

Anm. 21

So übereinstimmend Befürworter wie Kritiker der shame sanctions: Kahan (Anm. 2), S. $638 \mathrm{ff} ., 644 \mathrm{ff}$; Massaro, Michigan Law Review Vol. 89 (1991), S. 1880, 1890ff.; Pawlik (Anm. 12); Whitman (Anm. 2), S. 1062.

Siehe Schwerboff (Anm. 26), S. 165 mit entsprechenden Beispielen.

In diese Richtung auch Whitman (Anm. 2), S. $1089 \mathrm{f.}$

Massaro (Anm. 59), S. 1942 f.; dazu Whitman (Anm. 2), S. 1063. 


\section{a) Ehrenstrafen als Zugriff auf die Innerlichkeit?}

Ehrenstrafen sehen sich dem Vorwurf ausgesetzt, sie seien „strikingly illiber-

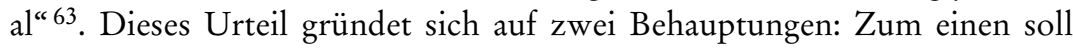
gerade der Vollzug von Schamstrafen offenbaren, dass dem Täter letztlich der Vorwurf gemacht werde, er habe die sozialethischen Normen der Gesellschaft nicht hinreichend verinnerlicht ${ }^{64}$. Zum anderen wird kritisch hinterfragt, ob die Strafe überhaupt auf einen „moralizing effect“ abzielen, also der Festigung der „moral values“ der Gesellschaft dienen dürfe ${ }^{65}$. Beide Vorwürfe kritisieren somit einen staatlichen Zugriff auf die Innerlichkeit: auf die des Täters bzw. die der Allgemeinheit.

Der erste Vorwurf attestiert der amerikanischen Rechtspolitik nicht weniger als die Aufhebung der Trennung von Moralität und Legalität. Diese Unterscheidung darf als ein wesentliches Erbe der Gedankenwelt der Aufklärung bezeichnet werden ${ }^{66}$. In der klassischen, auch für die Straftheorie relevanten Unterscheidung ${ }^{67}$ Kants bedeutet Legalität die von der Triebfeder des Handelns unabhängige, äußerliche Übereinstimmung einer Handlung mit dem Gesetz. Moralität ist demgegenüber die Qualitätsbezeichnung für eine Handlung, der als Triebfeder die Idee der Pflicht aus dem Gesetz zugrunde liegt ${ }^{68}$. Bloße Legalität ist damit zwar eine defiziente Gestaltung der inneren sittlichen Verfassung des Handlungssubjekts ${ }^{69}$. Für die Erfüllung rechtlicher Pflichten ist aber die äußerliche Normkonformität ausreichend, so dass - umgekehrt - nicht die ungenügende rechtsethische Einstellung, sondern der Verstoß gegen die Norm das allein zulässige Anknüpfungskriterium bildet ${ }^{70}$. Mehr als äußerliche Normkonformität wird aber auch von den shame sanctions nicht eingefordert: Indem sie an eine äußerliche Störung durch ein strafbares Verhalten anknüpfen, bestrafen sie (retrospektiv) die Gesinnung des Täters nicht in einem anderen, gar stärke-

63 Vgl. Kahan (Anm. 2), S. 596 m.w. N.

64 Vgl. Tonry (Anm. 33), S. 166.

65 Whitman (Anm. 2), S. 1089; siehe dazu auch Kaban (Anm. 2), S. 603.

66 Diese Unterscheidung wird mit Recht als „konfliktregulierendes Management“ der Moderne bezeichnet, siehe Kersting, Jean-Jacques Rousseaus Gesellschaftsvertrag, 2002, S. 69.

67 Siehe Schmitz, Zur Legitimität der Kriminalstrafe, 2001, S. 101.

68 Kant, Die Metaphysik der Sitten, 1797 (hier zitiert nach der Ausgabe von Ebeling, 1990), S. 53.

69 Kersting, Wohlgeordnete Freiheit, 1993, S. 178.

70 Kant (Anm. 68), S. 53; siehe auch Jakobs, Gedächtnisschrift für A. Kaufmann, 1989, S. $271,273 \mathrm{f}$. 
ren Ausmaß als alle anderen Strafen. Wie die Untersuchung des Kommunikationsprozesses gezeigt hat, erheben die shame sanctions (prospektiv) nicht einmal den Anspruch, an die Einsichtsfähigkeit des Täters zu appellieren und ihm die Norminhalte zu vermitteln. Vielmehr wenden sie sich in ihrer drastischen Symbolik an das Normbewusstsein der Betrachter. Damit setzen sie sich freilich, wie die positive Generalprävention insgesamt, dem Vorwurf aus, rechtsstaatswidrig eine rechtstreue Gesinnung durchsetzen zu wollen ${ }^{71}$. Freilich ist rechtssoziologisch anerkannt, dass das Strafrecht seine gesellschaftsund normstabilisierende Wirkung dann in optimaler Weise erfüllt, wenn es sich auf rechtsethische Überzeugungen der Gesellschaft stützen kann ${ }^{72}$. Aber auch normativ lassen sich die geltend gemachten Bedenken zerstreuen: Der (kantischen) Beschränkung des Rechts auf die Durchsetzung der Legalität des Handelns liegt nämlich die Pointe zugrunde, ein zwar äußerlich normkonformes Handeln genügen zu lassen, damit aber lediglich die Möglichkeit heteronomer Motivation neben die Chance autonomer, pflichtbewusster Motivation zu stellen. Indem das Exekutionsprinzip der Pflicht vom Zwang flankiert wird, soll die Gelegenheit zur anspruchsvolleren ethischen Gesetzgebung keineswegs verleugnet werden ${ }^{73}$. Anders gewendet: Das Verhalten allein an der Zwangsandrohung des Gesetzes auszurichten, ist legitim, führt aber nicht zu einer „Verminderung des Anspruchs der reinen praktischen Vernunft“ ${ }^{74}$. Zwar darf der Rechtszwang nicht an die Motivation geknüpft werden. Die Möglichkeit, über die Androhung und den Vollzug von Zwang auf die ethische Grundierung des Handelns Einfluss zu nehmen, wäre aber nur dann ausgeschlossen, wenn mittels des Strafvollzugs in einer Weise auf die Innerlichkeit der Betrachter eingewirkt würde, welche das intelligible Wesen des Menschen leugnete ${ }^{75}$. Das Ansprechen des Norm-

Mir Puig, ZStW 102 (1999), S. 915, 923: „Die Aufgabe des Strafrechts darf in einem die Menschenwürde achtenden Staat nicht darin bestehen, auf das sozialethische Bewußtsein der Bürger einzuwirken ...“; sowie a.a.O., S. 925: „Es geht nicht darum, die Verinnerlichung des Strafrechts zu autorisieren, weil dieses einem sozialen und demokratischen Rechtsstaat entstammt; dies würde einen krassen Widerspruch zu diesem Staatsmodell in sich enthalten, da der Staat eine rechtstreue Gesinnung seiner Bürger nicht gewaltsam durchsetzen darf. Es ist nicht zulässig zu strafen, um damit Rechtsbewusstsein zu erzeugen, sei dieses auch noch so progressiv“. Vgl. Würtenberger, Festschrift für Jescheck, Band 1, 1985, S. 37, 45.

73 Kersting (Anm. 69), S. 179.

74 Kersting (Anm. 69), S. 180.

75 Vgl. zu der tragenden Bedeutung der Anerkennung des intelligiblen Charakters des Menschen Kersting, Die politische Philosophie des Gesellschaftsvertrags, 1994, S. 184 f.; siehe auch Kühl, in: Jung/Müller-Dietz/Neumann, Recht und Moral, 1991, S. 139, 143. 
bewusstseins der Betrachter nimmt diesen also nicht per se ihre Dignität, vielmehr wird auf die Möglichkeit der Befreiung von der Fremdbestimmung hingewiesen: Führt nämlich der Weg von der ethischen Selbstverpflichtung zur rechtlichen Fremdverpflichtung in die Unfreiheit ${ }^{76}$, eröffnet die Anrufung der ethischen Gesetzgebung den Ausweg in die Freiheit. Wohlgemerkt: Das Recht bietet diese Möglichkeit, es verlangt nicht die Ergreifung derselben. Die aus den USA bekannt gewordenen Formen der shame sanctions mögen uns zwar verstören, sie sind aber nicht derart drastisch, dass der Schrecken der öffentlichen Strafvollziehung dem Betrachter die rechtsethische Gesinnung in einer Weise „einzubrennen“ suchte, welche ihm die Möglichkeit einer eigenen Vernunftentscheidung nähme. Die shame sanctions in ihrer heutigen Ausgestaltung sind kein „Fest der Marter“ mehr ${ }^{77}$.

Den Vorwurf eines unzulässigen Zugriffs auf die Innerlichkeit müssen sich die shame sanctions mithin nicht gefallen lassen.

\section{b) Shame sanctions als Bedrohung für die Gemeinschaft und ibre Friedensordnung?}

Immer wieder wird im Zusammenhang mit shame sanctions auch auf die Gefahr für die Gemeinschaft und ihren Frieden hingewiesen ${ }^{78}$. Dies schließt zwei Teilaspekte ein: Zum einen wird behauptet, die shame sanctions trügen den Keim der Auflösung der Rechtsgemeinschaft in sich. Zum anderen wird auf mögliche Friedensstörungen durch Übergriffe auf den Delinquenten abgehoben.

Die Tendenz zur Auflösung der Rechtsgemeinschaft wird den Ehrenstrafen attestiert, weil sie den Delinquenten dazu bringen könnten, sich von der rechtstreuen Gesellschaft ab- und Gruppen gleichfalls Exkludierter zuzuwenden ${ }^{79}$. Damit könnten Ehrenstrafen zu steigenden statt zu sinkenden Kriminalitätsraten beitragen ${ }^{80}$. Wenn aber shame sanctions einen Beitrag zur

76 Kersting (Anm. 69), S. 184.

77 Diesen Begriff für die öffentliche Hinrichtung des Mittelalters und der frühen Neuzeit verwendend Foucault (Anm. 9), S. 44.

78 Siehe Teichman (Anm. 29), S. 33; Whitman (Anm. 2), S. 1085, der allerdings meint, derartige Effekte müssten hingenommen werden, wenn der Vollzug von shame sanctions notwendig und legitim wäre.

79 Vgl. Whitman (Anm. 2), S. 1063.

80 Siehe Braithwaite, Crime, Shame and Reintegration, 1989, S. 89 u. S. 101, der aus diesem Grund ein „reintegrative shaming“ befürwortet, also „shaming which is followed by efforts to reintegrate the offender back into community of law-abiding or respectable citizens through words or gestures of forgiveness or ceremonies to decertify the offender 
Stabilisierung der Gesellschaft und ihrer Ordnung liefern sollen, indem mit ihnen die Verbindlichkeit bestimmter Normen betont wird, würden solche Folgen zweifellos dysfunktional wirken: Statt die Zentrifugalkräfte der Gesellschaft an die Rechtsordnung als festen Bezugspunkt zu knüpfen, könnte die Aufteilung der Gesellschaft in abgeschottete Gruppen befördert und ein Wettlauf in die „richtige“ Gruppe ausgelöst werden ${ }^{81}$. Tatsächlich führen shame sanctions eher dazu, bislang unverletzte Beziehungen des Täters zu seiner Umwelt zu beeinträchtigen ${ }^{82}$, statt die durch die Tat verletzten sozialen Beziehungen wiederherzustellen ${ }^{83}$. Diese Gefahr muss man freilich stets vor dem Hintergrund der Alternativen zu den Ehrenstrafen sehen. Stigmatisierung und Exkludierung sind nicht die ausschließliche Folge der shame sanctions, sondern werden in weit größerem Maße von einer Haftstrafe verursacht: Eine Freiheitsstrafe bedeutet nicht nur eine räumliche, sondern auch eine soziale Exklusion und führt bekanntlich dazu, dass sich der Inhaftierte eher mit der falschen Gruppe - seinen Mithäftlingen - identifiziert, als sich der Rechtsgemeinschaft anzuschließen. Selbst die kaum stigmatisierende Geldstrafe kann durch den Entzug von Vermögen zu einer Ausgrenzung aus dem bisherigen Lebensumfeld führen. Shame sanctions sind in dieser Hinsicht mithin ebenso ambivalent wie ihre Alternativen.

Konsequenzen für die Rechtsgemeinschaft resultieren somit nicht aus dieser Strafform, sondern sind Folge der rechtspolitischen Entscheidung, in welchem Ausmaß das Strafrecht eingesetzt werden soll.

In der Diskussion um die shame sanctions wird zudem auf die Gefahren für den inneren Frieden durch Übergriffe auf den Delinquenten verwiesen. Tatsächlich sind Friedensstörungen durch öffentliche Strafvollstreckungen nicht nur historisch belegt ${ }^{84}$, vielmehr trug eben jener Effekt entscheidend zum Ende des Prangers bei ${ }^{85}$. Es waren auch derartige Befürchtungen ${ }^{86}$, die

as deviant“ (S. 101). Damit fügt sich diese Sanktion als probates Mittel in eine restaurative Strafzweckkonzeption, so Vold/Bernhard/Snipes, Theoretical Criminology, Oxford, 2002, S. $306 \mathrm{f}$. Vgl. Teichman (Anm. 29), S. 31 ff.

Siehe dazu Friday, in: Kury/Obergfell-Fuchs (Hrsg.), Crime Prevention - New Approaches, 2003, 370, 371. Dieser Ansatz scheint in der Form mediativer Konfliktschlichtung auf große Zustimmung bei Tätern wie Opfern zu stoßen (a. a. O., S. 373). Foucault (Anm. 9), S. 78 ff.

85 Siehe dazu Whitman (Anm. 2), S. 1083; Foucault (Anm. 9), S. 85.

86 Dazu Freisler, in: Freisler/Luetgebrune (Hrsg.), Denkschrift des Zentralausschusses der Strafrechtsabteilung der Akademie für Deutsches Recht über die Grundzüge eines allgemeinen deutschen Strafrechts, 1934, S. 100, 113 f. 
zur Verwerfung des zur Zeit des Nationalsozialismus virulent gewordenen Gedankens beitrugen, Ehrenstrafen als zur völkischen Ideologie passende Sanktionsform einzuführen ${ }^{87}$. Shame sanctions könnten damit die tragende Säule jeder Straftheorie unterminieren: die Ermöglichung von gesellschaftlicher Ordnung und die Sicherung des inneren Friedens der Gesellschaft ${ }^{88}$. Ist die Friedenssicherung der wesentliche Grund für die Implementierung des Staates und seiner Institutionen, weil sich die Menschen entweder aus Klugheit den Gefahren der Rechtlosigkeit entziehen wollen ${ }^{89}$ oder weil sie aus Vernunft anerkennen, dass sie dem Leben einen selbstgesetzten Sinn nur in einer Rechtsgemeinschaft geben können ${ }^{90}$, müssen sich die Menschen in den Zustand allgemein anerkannter Rechtlichkeit begeben ${ }^{91}$. Der Staat hat folglich dieser Verpflichtung in zureichendem Maße gerecht zu werden, will er sich nicht in Widerspruch zu seiner Legitimationsgrundlage setzen. Vor dem Hintergrund dieser Verpflichtung ist die Gefahr zu sehen, dass sich die Reaktionen der Betrachter von Ehrenstrafen nicht in Empörung erschöpfen, sondern in Privatjustiz und handgreifliche Gewalt umschlagen.

Mit einer Bedrohung des inneren Friedens könnte aber ein Preis für die Strafe bezahlt werden, den selbst Kant nicht zahlen wollte: Zwar gilt sein üblicherweise zitiertes „Insel“-Beispiel ${ }^{92}$ als besonders eindrücklicher Beleg einer der Gerechtigkeit verpflichteten, das Ende eines Staates überdauernden Idee des Strafens ${ }^{93}$. Die Fokussierung auf diese Textpassage vernachlässigt

87 Da die Ehre für den nationalsozialistischen Staat ein ähnlich konstituierendes Gut darstellte wie die Freiheit und das Eigentum für den liberalen Staat, wurden Ehrenstrafen als probates Sanktionsmittel erachtet, siehe Dabm, DJZ 1934, 826f.; vgl. zur ehrbezogenen Straftendenz im NS-Staat Kubink, Strafen und ihre Alternativen im zeitlichen Wandel, 2002, S. $266 \mathrm{ff}$.

88 Statt vieler Jakobs (Anm. 7), 1. Abschnitt Rdn. 4; Jescheck/Weigend (Anm.18), S. 2; Jung, GA 1995, 535, 536; Maurach/Zipf, Strafrecht Allgemeiner Teil 1, 8. Aufl. 1992, $\$ 7$ Rdn. 1 ff.; Lackner/Kühl, StGB, 25. Aufl. 2004, \$46 Rdn. 1; auch Kaiser, Kriminologie, 3. Aufl. 1996, S. 312; siehe auch schon Durkheim, Über die Teilung der sozialen Arbeit, 1977, S. 149; Lubmann, Rechtssoziologie, 3. Aufl. 1987, S. 60 f., $106 \mathrm{ff}$.

Hobbes, Leviathan, 1651 (hier zitiert nach der Übersetzung von Mayer, Stuttgart 1970), 17. Kapitel; dazu Kersting, Thomas Hobbes zur Einführung, S. 146 f.

90 Kant (Anm. 68), S. 163.

91 Pawlik (Anm. 8), S. 81.

92 Siehe Kant (Anm. 68), S. 194f., wonach auch dann, wenn eine Gemeinschaft, die eine Insel bewohne, beschlösse, sich aufzulösen und die Insel zu verlassen, zuvor noch alle verurteilten Mörder hingerichtet werden müssten.

93 Umfassend dazu Zaczyk, in: Landwehr (Hrsg.), Freiheit, Gleichheit, Selbständigkeit. Zur Aktualität der Rechtsphilosophie Kants für die Gerechtigkeit in der modernen Gesellschaft, 1999, S. 73 ff.; zutreffend weist Altenhain, Gedächtnisschrift für Keller, 2003, S. 1, 11 f., darauf hin, dass die verurteilten Mörder im Inselbeispiel nicht allein 
aber eine im gleichen Kapitel vorgenommene Einschränkung, die den Gesamtkontext der kantischen Rechtslehre anschaulich macht: Führte die massenhafte Verurteilung von Mördern und ihren Komplizen dazu, dass der Staat bald keine Untertanen mehr habe und sich aufzulösen drohe, „d.i. in den noch viel ärgern, aller äußerer Gerechtigkeit entbehrenden Naturzustand“ überzugehen, müsse der Souverän von seinem Begnadigungsrecht Gebrauch machen ${ }^{94}$. Indem Kant sich in diesem Konfliktfall kollidierender Vernunftpflichten für die Pflicht zur Staatlichkeit entscheidet, muss er eine Ausnahme von der Verpflichtung auf die absolute Strafgerechtigkeit konstatieren und auf die - von ihm andernorts gescholtene ${ }^{95}$ - Begnadigung verweisen. Kants Beispiel zeigt freilich, dass Friedensstörungen der Vollziehung von Strafen erst ab einem Maß normativ entgegenstehen, welches Ehrenstrafen kaum je erreichen dürften: Das Postulat der absoluten Strafgerechtigkeit wird von seinem prominentesten Vertreter nämlich erst dann relativiert, wenn durch die Strafe eine Auflösung des Staates, dieser verstanden als gemeinsames Projekt zur Sicherung des Friedens und Ermöglichung von Gerechtigkeit, drohte ${ }^{96}$. Die shame sanctions opfern aber die Friedensordnung nicht auf den Richtplätzen der Moderne: Bei den bekannt gewordenen Übergriffen ${ }^{97}$ handelt es sich um Ausnahmen ${ }^{98}$. Ungeachtet solcher „statistischen“ Fragen bildet die Gefahr von Übergriffen auch kein prinzipielles Gegenargument. Ehrenstrafen erleichtern zwar die Identifizierung des Straftäters durch die Allgemeinheit und den Zugriff auf seine Person. Übergriffe und Aufruhr können aber durch staatliche Sicherungsmaßnahmen unterbunden und da-

einer „höheren Gerechtigkeit“ geopfert werden, sondern das Festhalten an der Idee der Gerechtigkeit das Verlassen der Insel überdauert und Grundlage der Staatlichkeit sein wird, in welcher die Inselbewohner ankommen werden.

Kant (Anm. 68), S. 196; siehe dazu aber Jakobs, Staatliche Strafe: Deutung und Zweck, 2004, S. 14, der dieses Ergebnis als „verwunderlich“ und „mit Kants System wohl nicht recht verträglich" bezeichnet.

96 Kant (Anm. 68), S. 196; zur Abhängigkeit der befriedenden Wirkung der Strafe von der Einordnung des Strafgesetzes in einen öffentlich-rechtlichen Zustand innerhalb der kantischen Rechtslehre siehe Zaczyk, Festschrift für Eser, 2005, S. 207, 219; siehe auch Hegel, Grundlinien der Philosophie des Rechts, 1820 (hier zitiert nach der Ausgabe von Lakebrink, 1970), $\$ 102$, der eine Strafe im Naturzustand als Rache bezeichnet, die ihrerseits Gegenreaktionen hervorrufe und sich ins Unendliche fortsetze.

Als vor einigen Jahren in New York der Vermieter eines rattenverseuchten Hauses in eben jenem Haus arretiert wurde, begrüßte ihn das wütende Gejohle der Menge („Welcome, you reptile!“), dazu Kahan (Anm. 2), S. 633. Schlimmer ging es dem Unschuldigen, der mit einem freigelassenen Sexualtäter verwechselt und von einem Mob angegriffen wurde, siehe Whitman (Anm. 2), S. 1088. 
mit die Friedensordnung gesichert werden. Auch hier ist es also keine Frage des „Ob“, sondern des „Wie“: In einem angemessenen Rahmen durchgeführt, führen Ehrenstrafen nicht zum Rückfall in die Lynchjustiz und zum Ende des innerstaatlichen Friedens.

\section{Die Strafe als wertbezogene und wertgebundene Reaktion}

Die in den USA vorgebrachten Argumente gegen die shame sanctions haben sich mithin als nicht durchschlagend erwiesen. Falls strafrechtstheoretische Gründe gegen die Ehrenstrafen sprechen, müssen diese tiefer in dem angelegt sein, was sich unter dem Begriff Strafe fassen lässt. Eine Antwort setzt also voraus, dass wir uns Klarheit über die Rechtsinstitution Strafe verschaffen.

\section{Wesenszüge der Reaktionsform „Strafe“}

Die Strafe hat im Laufe der Geschichte derart unterschiedliche Formen angenommen, dass immer wieder die Frage aufgeworfen wurde, ob es „die Strafe“ als ein über die Zeiten gleichbleibendes Gattungswesen überhaupt gebe ${ }^{99}$. Tatsächlich kann die Form der Strafe nicht ohne die Gesellschaft, nicht unabhängig von der Verfasstheit des Staates, gedacht werden. So kommt es, dass trotz einer - sowohl über die Zeit als auch die Grenzen hinweg bestehenden - Universalität der Vorstellung von Verbrechen signifikante Unterschiede bei den Sanktionsarten feststellbar sind ${ }^{100}$.

Ungeachtet ihrer wechselvollen Geschichte können aber typische Wesensmerkmale dessen beschrieben werden, was die Menschen intuitiv als Reaktion auf eine Straftat erwarten. Der Erwartungshaltung der Gesellschaft im Hinblick auf den Einsatz des Strafrechts Relevanz beizumessen, scheint freilich im Widerspruch zu der Prämisse zu stehen, dass die Strafe gesellschaftliche Erwartungen und Reaktionen formalisieren und kanalisieren soll ${ }^{101}$. Tatsächlich aber lässt sich die Bedeutung der gesellschaftlichen Erwartung für das Institut der Rechtsstrafe normativ begründen. Namentlich Hegel hat darauf hingewiesen, dass sich in der Strafe die Negation des nichtigen besonderen Willens des Täters und damit die Gültigkeit des allgemeinen Willens

99 Vgl. Stratenwerth/Kublen, Strafrecht Allgemeiner Teil, 5. Aufl. 2004, S. 3 f.

100 Kaiser (Anm. 88), S. 313.

101 Meier, Strafrechtliche Sanktionen, 2001, S. 6. 
manifestiere ${ }^{102}$. Der allgemeine Wille kann zwar durch das Verbrechen nicht verletzt werden. Indem sich der besondere Wille in der Tat aber Dasein gegeben hat, muss die nach außen getretene Desavouierung der Norm ${ }^{103}$ für unmaßgeblich erklärt werden. Diese äußerliche „Zeremonie“ der Strafe symbolisiert mithin, wer die Deutungshoheit über die Normen hat ${ }^{104}$.

Damit ist freilich nur begründet, weshalb sich auch die Strafe ein äußeres Dasein geben muss, nicht aber, weshalb für diesen Vollzug die Erwartungen der Gesellschaft normative Relevanz beanspruchen können. Dies wird erst bei Berücksichtigung der ganzen Bedeutung der Straftat deutlich: Da die vom Täter verletzte Rechtsordnung das Dasein aller in Freiheit sichert, wird mit der Straftat auch der durch die Rechtsordnung verbundene Bürgerverband angegriffen und das Loyalitätsansinnen der Mitbürger missachtet ${ }^{105}$. Die auf Bestrafung gerichtete Erwartungshaltung der Bürger an den Staat ist folglich nicht nur schlichte soziale Realität, sondern wegen jener Verletzung auch ihrer Rechte normativ begründbar. Daraus lässt sich jedoch immer noch nicht zwingend ableiten, dass der Staat dieser - legitimen - Erwartungshaltung mit einer Übelszufügung entsprechen muss, ja darf. Es mag zwar zutreffen, dass nur eine "harte Behandlung“ den in „unserem soziokulturellen Kontext üblichen Gebräuchen“ entspricht und daher die expressive Funktion des Tadels nur vermittels einer Übelszufügung erfüllt werden kann ${ }^{106}$. Damit ist aber der Bereich der Rechtssoziologie nicht verlassen und eine normative Begründung für die Legitimität nicht gefunden: Aus dem Sein folgt weder Sollen noch Dürfen. Eine Begründung für das Strafübel wird gelegentlich Kant zugeschrieben, der außerhalb seiner Rechtslehre festgestellt hat, dass alles Verbrechen Glückseligkeit wenigstens zum Teil verwirke ${ }^{107}$. Gegen eine Deutung des Strafübels als Ausgleich von Glückswürdigkeit und Glückseligkeit des Täters ${ }^{108}$ spricht aber nicht nur der Umstand, dass Kant den Gedanken in seiner Rechtslehre nicht aufgreift ${ }^{109}$. Vor allem ist nicht einzusehen, dass ausgerechnet die von Kant selbst immer

102 Hegel (Anm. 96), \97; vgl. auch Nagler (Anm. 3), S. 95.

103 Vgl. Jakobs (Anm. 7), 1. Abschnitt Rdn. $10 \mathrm{f}$.

104 Foucault (Anm. 9), S. 63.

105 Pawlik (Anm. 8), S. 86.

106 So etwa Hörnle, Tatproportionale Strafzumessung, 1999, S. 123.

107 Kant, Kritik der praktischen Vernunft, 1788 (hier zitiert nach der Ausgabe von Kopper, 1961), S. 65 f.

108 Siehe etwa Oberer, in: Brandt (Hrsg.), Rechtsphilosophie der Aufklärung, 1982, S. 399 , 401 ff; Welzel (Anm. 6), S. 238; kritisch Forschner, in: Brandt (a.a. O.), S. 376, $387 \mathrm{ff}$.; Jakobs (Anm. 94), S. 15.

109 Höffe, in: ders. (Hrsg.), Metaphysische Anfangsgründe der Rechtslehre, 1999, S. 221. 
wieder betonte individuelle Verschiedenheit und Zufälligkeit der Kategorie Glück ${ }^{110}$ als Maßstab für die Schaffung formaler Strafgerechtigkeit dienen soll ${ }^{111}$. Überdies kann man dem homo phaenomenon die Notwendigkeit einer Schmerzzufügung, einer Einbuße an Glückseligkeit individuell nicht vermitteln, sondern ihn nur, wie ein Instinktwesen, gefügig machen. Um sich der Legitimation der Übelszufügung zu nähern, muss man die individuelle Ebene verlassen. Stattdessen ist der Umstand in den Blick zu nehmen, dass die Verletzung der Rechtsordnung nicht mit der Wiedergutmachung des bei dem individuellen Opfer angerichteten Schadens vergolten werden kann, sondern die Strafe auch das Ausmaß an Illoyalität gegenüber dem gemeinsamen Bürgerprojekt eines „Friedens durch Recht“ abbilden muss ${ }^{112}$. Wird aber die Rechtsgemeinschaft durch die Tat verletzt, ist der an ihrer Freiheitsordnung entstandene Schaden in einer Weise zu vergelten, der von den Mitbürgern auch als Symbol für Strafe akzeptiert und entziffert wird ${ }^{113}$. Insofern ist es nicht nur legitim, sondern im System der Rechtsstrafe als Antwort auf die Verletzung des Loyalitätsansinnens der Mitbürger gleichsam angelegt, dass sich die Strafe besonderer Zeichen bedient, welche sie von anderen staatlichen Zugriffen auf den Einzelnen - etwa dem zivilrechtlichen Schadensersatz - abhebt ${ }^{114}$. Erst durch diese Sonderung von anderen staatlichen Maßnahmen wird der Allgemeinheit signalisiert, dass mit der Strafe nicht nur ein Schaden eines einzelnen ausgeglichen, sondern eine Verletzung

110 Kant (Anm. 107), S. 45 ff.

111 Döring, Feuerbachs Straftheorie und ihr Verhältnis zur Kantischen Philosophie, 1907, S. 10, weist auf den „Widerstreit zwischen der uns unmittelbar und unabweisbar von der praktischen Vernunft gegebenen Idee der Strafwürdigkeit jeder sittlichen Übertretung und dem empirisch aufgegriffenen Prinzip der Glückseligkeit" hin und deutet die Textstelle als bloße Ablehnung des Eudämonismus ohne Anspruch auf Legitimierung der Strafe.

112 So Pawlik (Anm. 8), S. 92.

113 Die symbolische Komponente der Strafe wird denn auch weithin anerkannt, siehe etwa Bock, ZStW 103 (1991), S. 636; Feinberg, in: Duff/Garland (Anm. 37), S. 71, 74; Kahan (Anm. 2), S. 592 ff.; Kaiser (Anm. 100), S. 312; Kunz (Anm. 14), \ 39 Rdn. 33; Meier (Anm. 101), S. 5 f.; Rössner, Bewährungshilfe, 1994, S. 18, 24.

114 Vgl. Pawlik (Anm. 8), S. 91, der eine gewisse Drastik der strafenden Sanktion als normativ angemessene Antwort auf die Anerkennungsverletzung erachtet; Müssig, Schutz abstrakter Rechtsgüter und abstrakter Rechtsschutz, 1994, S. 146, begreift das Strafübel als „Produkt normativer Verständigung“; Jakobs, Norm, Person, Gesellschaft, 2. Aufl. 1999, S. 104f., stellt darauf ab, dass die Strafe mit der Wegnahme von gesellschaftlichen Interaktionsmitteln „die Wirklichkeit der nur-formellen Person in der Tat, also den Normbruch,“ mit der „Wirklichkeit der Norm“ konfrontiere; ders. (Anm. 94), S. 29, wonach sich der Schmerz der Strafe mit der „kognitiven Sicherung der Normgeltung“ als Zweck der Strafe erkläre. 
der Regeln ihres Zusammenlebens vergolten wird. Diese besondere Zeichenfunktion erfüllt die Übelszufügung, das „,hard treatment“ ${ }^{\text {“115. }}$.

Fasst man all dies zusammen, ist die Strafvollziehung Ausdruck der Vergeltung einer Verletzung des Rechts als Recht, welche sich, da auf Kommunikation in die mitverletzte Bürgergemeinschaft angelegt, einer übelszufügenden Symbolik bedienen darf, die von der Gesellschaft als tadelnd identifiziert wird.

\section{Strafe als wertbezogene und wertgebundene Institution}

Freilich muss die Strafe und damit die Strafform im Rechtsstaat den Anspruch erfüllen, eine Rechtsstrafe und damit mehr zu sein als ein bloßes Symbol, mit welchem signalisiert wird, hier werde einer - legitimen - gesellschaftlichen Erwartung entsprochen. Die Strafe ist nach einem treffenden Vergleich Harts eine soziale Institution wie das Eigentum, die ihre wesentliche Struktur durch das Recht empfängt ${ }^{116}$. Sie ist eine wertbezogene und wertgebundene Reaktion.

Der Wertbezug ist dem Tadel immanent, bezieht er sich doch auf den Verstoß gegen eine bestimmte Norm und missbilligt diesen. Fände die Strafe ihre rechtliche Struktur aber allein in diesem Wertbezug, könnte das Strafrecht aus einem breiten Arsenal geeigneter Strafformen schöpfen. Die Ehrenstrafen mit ihrer symbolischen Missbilligung müssten als gleichsam optimales Instrument öffentlichen Tadels propagiert werden. Schon Kant hat aber die von ihm aufgeworfene Frage, ob Art und Maß der Strafe dem Gesetzgeber gleichgültig sein könnten, solange sie nur ihr Ziel erreiche, mit Betonung des Wiedervergeltungsrechts als a priori gültigem Prinzip des Strafrechts verneint ${ }^{117}$. Damit wird freilich nur die innersystematische Proportionalität im Sinne einer Abstufung der Strafe nach der Schwere des

115 Abweichend Kühl, Die Bedeutung der Rechtsphilosophie für das Strafrecht, 2001, S. $32 \mathrm{f}$., der das Strafübel maßgeblich mit der den Täter adressierenden Missbilligung legitimiert. Jedenfalls beruht die Übelszufügung nicht auf einem „Zufall“ (so aber Günther, Festschrift für Lüderssen, 2002, S. 205, 219); auch kann die Demonstration der Normgeltung nicht ohne weiteres durch andere, nicht-strafende Mittel ersetzt werden, so aber Hörnle/v. Hirsch, GA 1995, 261, 266; Mir Puig, ZStW 102 (1990), S. 914, 922 f.; anders schon Nagler (Anm. 3), S. 95.

116 Siehe Hart, Punishment and Responsibility, Oxford, 1968, S. 3: „In both cases [Eigentum und Strafe, der Verf.] we have to do it with a social institution of which centrally important forms is a structure of legal rules ..."; siehe auch Duff/Garland, in: dies. (Anm. 37), S. 23.

117 Kant (Anm. 68), S. 230 f. 
jeweiligen Deliktes verlangt ${ }^{118}$. Fragt man hingegen nach der zulässigen Schwere und der Form einer Strafe, etwa für einen Diebstahl oder einen Mord, und nimmt das kantische Talionsprinzip beim Wort ${ }^{119}$, gewährt es, mit den Worten Nietzsches, ein Recht auf Grausamkeit ${ }^{120}$ : Soll der Mörder sterben und kann der Dieb versklavt werden, erhält die Strafe in der Tat die „Physiognomie des Verbrechens" ${ }^{121}$. Andererseits muss das ius talionis nicht als spezifische Vergeltung, sondern kann auch als Wiedervergeltung der Form und dem Wert nach verstanden werden ${ }^{122}$ : Wenn sich der Dieb auch selbst bestiehlt, der Mörder sich auch selbst tötet, wie Kant schreibt ${ }^{123}$, dann vollzieht eine nach „Qualität und Quantität“ 124 an der Tat orientierte Strafe nur das nach, was vom Täter bereits in seiner Tat postuliert worden ist. Kant selbst hat freilich diesen Weg nicht ausdrücklich aufgezeigt ${ }^{125}$. Er beließ es vielmehr bei dem ius talionis als Kriterium „formaler Korrespondenz“126. Innerhalb der Grenzen einer solchermaßen proportionalen Strafe kann dann nach dem „Nutzen für ihn [den Täter] selbst und seine Mitbürger“ gefragt und mithin Strafklugheitserwägungen Raum gegeben werden ${ }^{127}$. Doch auch hier bleibt Kant dem Leser nähere Hinweise schuldig.

Es ist Hegel zu verdanken, die bei Kant angelegten Gedanken - Vergeltung der Tat ihrem Wert nach, Trennung von Strafgerechtigkeit und Strafklugheit - weiter ausgearbeitet und sie in ein umfassendes System der Straftheorie eingebunden zu haben: Die Aufhebung des Verbrechens ist danach insofern Wiedervergeltung, als sie dem Begriff nach Verletzung der Ver-

118 Welzel (Anm. 6), S. 241; Höffe (Anm. 109), S. 213, 228.

119 Siehe Hegel (Anm. 96), $\mathbb{\$} 101:$,[...] es ist sehr leicht, die Wiedervergeltung der Strafe (als Diebstahl um Diebstahl, Raub um Raub, Aug um Aug, Zahn um Zahn, wobei man sich vollends den Täter als einäugig oder zahnlos vorstellen kann) als Absurdität darzustellen, mit der aber der Begriff nichts zu tun hat, sondern die allein jener herbeigebrachten spezifischen Gleichheit zuschulden kommt" (Hervorhebung im Original).

120 Nietzsche, Zur Genealogie der Moral, 1896 (hier zitiert nach der Ausgabe von Gerhardt, 1988), Abschnitt 5.

121 Berner, Lehrbuch des deutschen Strafrechts, 1876, S. 30.

122 So Höffe (Anm. 109), S. 227. Dieser verweist auf Kant (Anm. 68), S. 363: „[...] das ius talionis der Form nach noch immer für die einzige a priori bestimmende [...] Idee des Strafrechts“. Und weiter a.a. O.: „Nur dann kann der Verbrecher nicht klagen, daß ihm Unrecht geschehe, wenn er seine Übeltat sich selbst über den Hals zieht und ihm, wenngleich nicht dem Buchstaben, doch dem Geiste des Strafgesetzes gemäß widerfährt, was er an anderen verbrochen hat"; siehe auch Jakobs (Anm. 94), S. 16.

123 Kant (Anm. 68), S. 194.

124 Kant (Anm. 68), S. 193.

125 So Höffe (Anm. 109), S. 227.

126 Höffe (Anm. 109), S. 228.

127 Kant (Anm. 68), S. 192; dazu auch Schild, ARSP 70 (1984), S. 71, 85 f. 
letzung ist, so dass die Vergeltung des Verbrechens durch die Strafe dem Begriff nach keine spezifische Gleichheit, sondern nur eine dem Wert gemäße verlangt ${ }^{128}$. Da aber die qualitative und quantitative Beschaffenheit des Verbrechens und seiner Aufhebung durch die Strafe in die Sphäre der Äußerlichkeit falle, die keiner absoluten Bestimmung, sondern nur einer Annäherung zugänglich sei ${ }^{129}$, kann und will Hegel hier keine konkreten Vorgaben für die Art und das Maß der Strafvollstreckung machen. Auf dieser, dem Substantiellen der Strafe nachgelagerten Ebene ${ }^{130}$ der Sanktionen und ihrer Vollziehung soll sich der Gesetzgeber daher am gesellschaftlichen Entwicklungsstand orientieren dürfen ${ }^{131}$ : Zur Erfüllung seiner friedenssichernden Aufgabe stehen ihm folglich die Normen und Sanktionen zur Verfügung, die im Verhältnis zur Verfasstheit der Gesellschaft angemessen und mit dem gesamten Kulturzustand des Volkes und den Rechten des Individuums vereinbar sind ${ }^{132}$. Koppelt man den zulässigen Inhalt von Strafnormen und Sanktionsarten derart an den gesellschaftlichen Entwicklungsstand und seine praktischen Erfordernisse ${ }^{133}$, steht dem Staat ein großes Arsenal zulässiger, weil funktionaler Strafen zur Verfügung. Dem Staat und dem Strafrecht ein solches Instrumentarium an Normen und Sanktionen zur Verfügung zu stellen, heißt freilich nicht, ohne gesellschaftliche Notwendigkeit den Schutz vor Kriminalität mit der Freiheit der Individuen erkaufen zu wollen ${ }^{134}$. Damit der Staat und das Strafrecht nicht die Subjekte, zu deren Schutz sie angetreten sind ${ }^{135}$, verschlingen, darf das bürgerschaftliche Projekt „Frieden durch

128 Hegel (Anm. 96), $\$ 101$.

129 Hegel (Anm. 96), \$101. Aus der Wertvergeltung folgt, dass sich die Gleichheit der Vergeltung nicht auf die „äußere spezifische Gestalt“ beziehen dürfe, so Hegel (a.a.O.), womit der Forderung Berners (siehe Anm. 121) Genüge getan ist.

130 Hegel (Anm. 96), $\$ 101$.

131 Hegel (Anm. 96), $\$ 96(Z), \mathbb{} 218(Z) ;$ Abegg, Die verschiedenen Straftheorien in ihrem Verhältnis zu einander und zu dem positivem Rechte und dessen Geschichte, 1835, $\int 19$.

132 So Jescheck/Weigend (Anm. 18), S. 3; siehe auch Bockelmann, Einführung in das Recht, 1975, S. 38 ff., insbes. S. 40.

133 Abegg (Anm. 131) \21; Hegel (Anm. 96), \218; Jescheck/Weigend (Anm. 18), S. 3; anders Nietzsche (Anm. 120), S. 311, der „den Brauch, den Akt, das ,Drama“" als das „reaktiv Dauerhafte“ und demgegenüber „das Flüssige an ihr, den Sinn, den Zweck, die Erwartung“ nennt; ähnlich Durkheim (Anm. 88), S. 128, der die „Natur der Strafe“ als gefestigt und unabhängig von den ihr zugewiesenen Strafzwecken erachtet.

134 Siehe auch Jakobs (Anm. 7), 1. Abschnitt Rdn. 13 b.

135 Siehe dazu schon Köstlin, System des deutschen Strafrechts, Allgemeiner Teil, 1855, S. 395: „Diese Zweckbestimmungen [der Strafe, der Verf.] haben nun ihre Berechtigung in der Einsicht, daß das Objektivsittliche (das Recht), wie alles Sittliche, durch Freie und für Freie gesetzt sei, mithin das Individuum nicht bloß als Accidenz des Staats be- 
Recht“ ${ }^{136}$ nicht durch eine Terrorherrschaft gesichert werden ${ }^{137}$. Folglich ist die Strafe in dem Sinne wertgebunden, als sich in ihr die Durchsetzung des „Rechts als Recht, dieses verstanden als Daseinsordnung der Freiheit“ ${ }^{138}$ manifestieren muss. Sie muss ,jene regulativen eusozialen-ethischen Funktionen besitzen, welche die asozial-unethischen Funktionen des Unrechts aufheben“ 139 .

\section{Folgen der Wertgebundenheit der Strafe}

Aus diesem Verständnis der Strafe als Institution zur Durchsetzung des Rechts als Recht lassen sich für den hier interessierenden Zusammenhang mehrere Ausprägungen der Wertbindungen ableiten, deren Grenzen auch von Ehrenstrafen nicht gesprengt werden dürfen.

\section{a) Die Bindung der Strafe an den verfassungsrechtlichen Regelrabmen}

Das Strafübel verfehlt seine Aufgabe der Manifestation des status quo ex ante, wenn nicht nur das vom Täter negierte Recht als gültig bestätigt wird, sondern die Strafvollstreckung eine darüber hinaus greifende Botschaft vermittelt, die sich nicht mit dem vorgängig-allgemeinen Recht vereinbaren lässt. Wenn nämlich die Strafe lediglich Rückwirkung der Tat ist, muss sie nicht nur in ihrer konkreten Erscheinungsform, nach Art und Maß einen der Tat entsprechenden Wertumfang haben ${ }^{140}$. Vielmehr muss sie ihrem Regelrahmen entsprechen, in welchen das Strafrecht mit seinen Sanktionen eingefügt ist und dessen Grenzen es zu beachten hat, soll es nicht zu einer Negation des Rechts gerade dadurch kommen, dass das vom Täter negierte Recht wiederhergestellt wird. Anders gewendet: Die Art der Vollstreckung der Strafe darf keine auf Allgemeinheit Anspruch erhebende teilweise Negation des Allgemeinen sein. Somit ist die Auffassung Naglers, die Funktion der

trachtet werden, vielmehr das Recht auch um des Menschen willen, für ihr Wohl dasein müsse, - eine Anerkennung, die am wenigsten in der Strafrechtspflege fehlen darf, da diese den Menschen in seinem Innersten, im freien Willen selbst ergreifen.“.

136 Pawlik (Anm. 8), S. 90.

137 So Schmitz, Zur Legitimität der Kriminalstrafe, 2001, S. 137, in Bezug auf die Staatstheorie von Hobbes.

138 Pawlik (Anm. 8), S. 87.

139 So Lampe, in: Jung/Müller-Dietz/Neumann (Hrsg.), Recht und Moral, 1991, S. 305, 307, dabei die Notwendigkeit einer Sühneleistung betonend.

140 Hegel (Anm. 96), $\mathbb{S}$ 96; siehe dazu Mohr, in: Höffe (Hrsg.), Hegel, Grundlinien einer Philosophie des Rechts, 1997, S. 95, 117; Müller-Tuckfeld, Integrationsprävention, 1998, S. 277. 
Strafe bestimme wesentlich das Mittel ${ }^{141}$, insofern verkürzt, als dies den wesentlichen Umstand der Rechtsstrafe vernachlässigt, in ihrer Besonderheit die Grundprinzipien des Allgemeinen enthalten zu müssen ${ }^{142}$. Dies verdeutlicht, dass für das Rechtsinstitut Strafe die Beachtung des verfassungsrechtlich geschützten allgemeinen Persönlichkeitsrechts nicht bloß Ausfluss eines rechtstheoretischen Anwendungsvorranges ist. Vielmehr ist die Einbeziehung der Wertungen des Regelrahmens unmittelbar in der Wertgebundenheit der Institution Strafe angelegt. Freilich ist damit nur soviel an Wert gewonnen, wie der verfassungsrechtliche Regelrahmen versprechen kann. Indem die Vollstreckung der Strafe abhängig von den Wertungen der Verfassung ist, die ihrerseits nur Ausdruck des „Kulturzustandes“ der Gesellschaft sind, wird deutlich, dass Hegels Hinweis auf die gesellschaftliche Relativität der Sanktionen auch unter der Geltung des Grundgesetzes Berechtigung hat: Sollte nämlich die stigmatisierende Wirkung von Ehrenstrafen angesichts des gesellschaftlichen Entwicklungsstands als notwendig zur positiven Generalprävention erachtet werden, stellte dies ein verfassungsrechtliches Interesse dar, mit welchem das allgemeine Persönlichkeitsrecht des Verurteilten für eine Abwägung geöffnet werden könnte ${ }^{143}$.

141 Nagler (Anm. 3). S. 99; anders Androulakis, ZStW 108 (1996), S. 300, 305.

142 Vgl. Hegel (Anm. 96), \101; die Wiederherstellung des Rechts durch die Strafe vermag damit auch die Forderung systematisch stringent zu erklären, die positive Generalprävention habe sich über die Normen des Strafrechts hinaus an Grund- und Verfahrensrechten zu orientieren, dazu namentlich Hassemer, in: Hassemer/Lüderssen/ Naucke (Hrsg.), Hauptprobleme der Generalprävention, 1979, S. 23, 53 f.: „Die soziale Kontrolle abweichenden Verhaltens durch das Strafrecht ist in spezifischer Weise gebunden, formalisiert: Zur Gewährleistung elementaren Grundrechtsschutzes des Abweichenden ist die Kontrollinstanz Strafrechtssystem an Normen gebunden“. Dazu auch ders., Festschrift für Lüderssen, S. 205, 230f.; ders., in: Schünemann/v. Hirsch/ Jareborg (Hrsg.), Positive Generalprävention, 1998, S. 29, 44 f.

143 Wer die abwägende Rechtsprechung des BVerfG liest, wird es nicht als ausgemacht ansehen, dass Ehrenstrafen in Situationen sich ausbreitender Kriminalität zwingend als verfassungswidrig verworfen würden. Das BVerfG hält etwa die aus einer Veröffentlichung des Namens und Bildes resultierende stigmatisierende Wirkung nicht für grundsätzlich verfassungswidrig, sondern wägt das Persönlichkeitsrecht mit anderen Verfassungsgütern ab, siehe BVerfGE 35, 202, $219 \mathrm{ff}$. Auch eine „Prangerwirkung“ von Berichten über Straftaten ist zumindest beim Vorliegen gewichtiger Gründe als hinnehmbar bezeichnet worden (BVerfGE 97, 391, 404ff.). Siehe zum Verbot der unmenschlichen und erniedrigenden Strafe nach Art. 3 EMRK auch EGMR Vol. 60 1997, VIII, S. 2804, 2822, wo die Öffentlichkeit des Strafaktes zumindest als „relevant factor“ für die Beurteilung nach Art. 3 EMRK bezeichnet wird. 


\section{b) Das Rechtssubjekt als Adressat der Rechtsstrafe}

Soll Recht als Bürgerprojekt zur Freiheitsgewährleistung durchgesetzt werden, muss die Strafe den Delinquenten als Bürger behandeln, sie muss ihm als Vernünftigen vermittelbar sein. Auch wenn man anerkennt, dass der Umgang mit dem Delinquenten als Ausfluss des Kulturzustandes des Volkes von der gesellschaftlichen Entwicklung abhängig ist, hat diese Entwicklung solange einen Fixpunkt, als man an dem Postulat festhält, nach welchem Recht als Recht durchgesetzt werden soll: Jede mit der gesellschaftlichen Entwicklung einhergehende Veränderung der Rechtsstrafe muss an dem Punkt enden, an welchem die Strafe nicht mehr an einem Rechtssubjekt, dem Bürger, vollstreckt wird, sondern am bloßen Individuum, dessen Vernunftbegabung für die Bestrafung keine Voraussetzung mehr ist ${ }^{144}$. Allein die mit der Strafe verbundene Zufügung von Übeln macht die Strafe für den Delinquenten freilich nicht sinnwidrig, solange ihm trotz der mit ihr verbundenen Einbuße an Glückseligkeit die Einsichtsmöglichkeit belassen wird, dass er durch seine Straftat vernunftwidrig gehandelt und dafür die Folgen zu tragen hat ${ }^{145}$. Ein solch emphatisches Strafverständnis schließt Strafformen aus, die vom Delinquenten nicht als Mittel zur Durchsetzung des Rechts, sondern als Ausdruck der Willkür oder Rache erkannt werden müssen ${ }^{146}$. Die Vermittelbarkeit einer vom Individuum als Rechtsverlust erlebten Strafauferlegung ist daher wesentlich an das Verfahren gebunden: Die Auferlegung des Strafübels würde unvermittelt zurückgewiesen, wenn der Verurteilung und der Vollstreckung schon die Förmlichkeiten als „dem Recht nicht äußerliche, sondern notwendige Zeichen der Anerkennung“ fehlten ${ }^{147}$. Der intuitiven Ablehnung zum Trotz führen Ehrenstrafen indessen nicht auf den Weg zurück zu einer willkürlich rächenden, archaischen Strafe am Individuum. Gewiss stellen sie ein „hard treatment“ dar, eine un-

144 Siehe Jakobs (Anm. 114): „Der Normbrecher muß Person bleiben“. Die von Foucault (Anm. 9), S. 21, beschriebene Entwicklung der Strafvollstreckung am „wirklichen, schmerzempfindlichen Körper“ hin zur Vollstreckung am ,juristischen Subjekt“, die in der Abschaffung grausamer Hinrichtungsarten durch die Einführung der Guillotine zum Ausdruck komme, ist im System der Rechtsstrafe mithin unumkehrbar.

145 Köbler, Der Begriff der Strafe, 1986, S. 36; Abegg (Anm. 131), \22. Dass sich das (auch individuell) Gute nicht mit der individuellen Glückseligkeit gleichsetzen lässt, hat schon Sokrates bewiesen, siehe dazu Schmitz (Anm. 137), S. $80 \mathrm{ff}$.

146 Abegg (Anm. 131), $\mathbb{8}$; siehe auch Hegel (Anm. 96), $\$ 102$.

147 Vgl. Müller-Tuckfeld (Anm. 140), S. 279f.; zur verfassungsrechtlich verlangten Förmlichkeit von Strafverfahren und Strafvollzug Hassemer, Festschrift für Lüderssen, S. 205, 238 f.; BVerfGE 33, 1, 11. 
menschliche Behandlung sind sie aber nicht ${ }^{148}$ : Den wesentlichen Unterschied zu den gängigen Strafformen stellt die Tatsache dar, dass sie die Diskretion des Strafvollzugs beenden und den Delinquenten mitsamt seinem Vergehen an die Öffentlichkeit ziehen. Die stigmatisierende Wirkung der Strafe ist bei shame sanctions folglich augenfälliger als bei anderen Strafen ${ }^{149}$. Doch hieße es, den stigmatisierenden Charakter anderer Strafformen zu leugnen, wenn man in den Ehrenstrafen etwas qualitativ Neues erblicken wollte: Entlassene Häftlinge werden sich Fragen aus ihrer Umgebung nach dem Grund ihrer Abwesenheit stellen müssen. Auch die rechtlichen Folgen der Tat sind mit der Strafverbüßung keineswegs „abgewickelt“, schließt sich an das Strafverfahren doch zumeist ein Zivilverfahren mit der Vollstreckung des entsprechenden Urteils an. Straftäter können also weder tatsächlich noch rechtlich verlangen, nach der Strafverbüßung nicht mehr mit der Tat konfrontiert zu werden ${ }^{150}$. Das Stigma der Strafe ist letztlich Folge der als notwendig und legitim erkannten Zeichenwirkung der Übelszufügung. Kommt eine Strafe aber ohne dieses „hard treatment" nicht aus, wäre ein kategorisches Argument gegen die Ehrenstrafen nur dann gefunden, wenn die stigmatisierende Wirkung ein Ausmaß erreichte, welches den Delinquenten außer Stande setzte, nach der Strafverbüßung wieder Teil der Gesellschaft und damit Teil ihrer Friedens- und Rechtsordnung zu sein ${ }^{151}$. Dies ist allenfalls im Hinblick auf die mit dem Begriff „Megan’s Law“ bezeichneten Fälle vorstellbar, in denen Menschen nach der Strafverbüßung der Nachbarschaft als Kinderschänder oder Vergewaltiger vorgestellt werden. Hier wird es dem Delinquenten gerade unmöglich gemacht, durch einen Umzug seine Vergangenheit hinter sich zu lassen und unbelastet von ihr in der Gesellschaft einen Platz finden zu können. Freilich: Begreift man diese Kennzeichnungspflicht als eine Form der Maßregel der Sicherung, dürfte deutschen Betrach-

148 Es lässt sich daher nicht pauschal sagen, shame sanctions seien eine grausame, die Würde verletzende Strafform, siehe aber Massaro (Anm. 59), S. 1942f.; vgl. auch Whitman (Anm. 2), S. 1069, der meint, dass diesem Einwand „intuitive Plausibilität“ zukomme, er allein aber inadäquat sei, um zu erklären, „what is wrong with the shame sanctions".

149 Vgl. zur Wirkung einer Stigmatisierung auf den Status einer Person Page, Stigma, London 1984, S. 10: „The individual [...] is assigned a ,master status trait': homosexual, drug addicted, prostitute, juvenile delinquent, or others [...] this label will dominate all other ,characteristics' of the individual: ,good athlete', ,good conversationalist', good dancer" “.

150 Deutlich BVerfG NJW 2000, 1859f. „Das allgemeine Persönlichkeitsrecht vermittelt Straftätern aber keinen Anspruch darauf, in der Öffentlichkeit überhaupt nicht mehr mit der Tat konfrontiert zu werden“.

151 Pawlik (Anm. 8), S. 94 f. 
tern das Kopfschütteln schnell vergehen: Die Antwort unseres Sanktionensystems wäre im Zweifel die Wegsperrung eines prospektiv als gefährlich erkannten Täters ${ }^{152}$. Soll der Staat den Frieden der Bürger garantieren, bleibt sein Rechtssystem keineswegs auf die an eine Straftat anknüpfende Reaktion beschränkt. Vielmehr ist er zur Aktion gegen einen Menschen berechtigt, welcher die Mitbürger bereits durch die "Gesetzlosigkeit seines Zustands“ lädiert ${ }^{153}$. In Anbetracht dessen kann von einem Tabubruch durch die Ehrenstrafen jedenfalls in dieser Hinsicht keine Rede sein.

Das Gebot menschengerechter Behandlung wäre freilich verletzt, wenn mit dem bekannten Einwand Kants gegen die relativen Straftheorien - der Delinquent zum Mittel degradiert würde ${ }^{154}$. Eine derartige Verletzung durch die Ehrenstrafen liegt jedenfalls nicht in der Verurteilung zur passiven Duldung der Betrachtung: Zwar wird die komplexe soziale Tatsache „Straftat“ mitsamt ihrer Umstände, welche bereits im Strafverfahren auf das rechtlich Wesentliche verkürzt wurde ${ }^{155}$, in der Vollziehung einer shame sanction vollends auf ein Symbol gebracht. Eine solche Reduktion des tatsächlichen Geschehenen ist freilich die zwangsläufige Folge jeder Strafe. Zum Mittel würde der Delinquent indessen dann, wenn der Pranger und damit der Angeprangerte allein als Instrument zur Einwirkung auf das Normbewusstsein der Betrachter eingesetzt und die Stigmatisierung zum Zweck der Bildung einer rechtstreuen Gesinnung der Bevölkerung hingenommen würde ${ }^{156}$. Begreift man aber die Strafe als Vergeltung für die Tat, wird der Täter eben nicht als bloßes Mittel der Generalprävention herangezogen: Seine primäre Verpflichtung zur Erbringung von Loyalität gegenüber der Rechtsordnung der Bürger wandelt sich zu einer sekundären Pflicht, die Bestätigung des unauflöslichen Zusammenhangs von Freiheitsgenuss und Loyalität auf seine Kosten zu dulden ${ }^{157}$. Die Pflicht zur Duldung der Normmanifestation durch eine Ehrenstrafe enthält insoweit keinen qualitativen Unterschied im Vergleich zur Rechtsbestätigung durch die Anwendung anderer Strafen. In einer schuldangemessenen Weise eingesetzt, konfligieren Ehrenstrafen folg-

152 Treffend spricht Jakobs (Anm. 94), S. 40, von einer „partiellen Entpersonalisierung“ durch derartige Maßregeln.

153 Vgl. Kant, Zum ewigen Frieden, 2. Aufl., 1796, (zitiert nach der Weischedel-Ausgabe, 1977, Band 11), S. 203.

154 Kant, Kritik der praktischen Vernunft, S. 45, 132.

$155 \mathrm{Vgl}$. Ellscheid/Hassemer, in: Lüderssen/Sack (Hrsg.), Seminar: Abweichendes Verhalten, Band II, 1975, S. 266, 275; siehe dazu Hörnle/Hirsch, GA 1995, 261, 273 f.

156 Vgl. Hassemer (Anm. 142 ), S. $40 \mathrm{f}$.

157 So Pawlik (Anm. 8), S. 90 f.; siehe auch Jakobs, ZStW 107 (1995), S. 843, 844. 
lich nicht mit dem Verbot, den Bürger auch in der Strafe nicht zum Mittel herabzustufen.

\section{c) Strafoollzug durch Rechtsinstitutionen}

Aus dem Verständnis der Strafe als wertbezogener und vor allem wertgebundener Institution leitet sich schließlich ein weiteres Postulat ab: Die Strafe muss nicht nur am Bürger vollzogen werden. Sie darf - gleichsam auf der anderen Seite - nicht von Individuen, sondern muss von Institutionen vollstreckt werden, welche die Bürgergemeinschaft dafür auserkoren hat. Diese Institutionen müssen gewährleisten, dass der Gesellschaft der Unterschied zwischen durchzusetzendem Recht und missbilligtem Unrecht kenntlich gemacht wird: Es sind daher Strafformen und Vollstreckungsarten zu vermeiden, die eine qualitative Nähe zum Verbrechen haben ${ }^{158}$. Zudem muss die Förmlichkeit des Vollstreckungsverfahrens verdeutlichen, dass die Folge von Straftat und Strafe keine „unvernünftige Sequenz zweier Übel“ ist ${ }^{159}$, sondern sich Unrecht und Recht gegenüber stehen ${ }^{160}$. Zwar ziehen auch konventionelle Strafen informelle Reaktionen nach sich, sei es durch die Reaktionen anderer Gefängnisinsassen auf bestimmte Delinquenten (Vergewaltiger, Ausländer ect.), sei es durch das Gespött Böswilliger nach der Strafverbüßung. Im Gegensatz zu diesen Nebenfolgen der eigentlichen Strafe durchbrechen Ehrenstrafen indessen die für staatliche Reaktionen kennzeichnende Förmlichkeit schlechthin: Die informellen Reaktionen der Betrachter sind keine Kollateralschäden der eigentlichen Strafe, sondern das Strafübel selbst. Indem shame sanctions sich mithin entscheidend auf Verhaltensweisen von Individuen stützen, die nicht als spezifische Antwort für Rechtsverletzungen reserviert sind ${ }^{161}$, bilden sie ein Konglomerat aus normbezogener staatlicher Sanktion einerseits und von Schaulust, Schadenfreude oder gar Rache geleiteten Reaktionen der Bevölkerung andererseits. Die Förmlichkeit des Strafens als Kennzeichen staatlicher Rechtsdurchsetzung wird nicht nur gezielt verwässert. Der Staat macht sich bei der Vollstreckung

158 Ein solcher Bezug besteht, wenn im Virginia der Kolonialzeit einem Mehldieb die Zunge durchbohrt und dieser anschließend angekettet dem Hungertod überlassen wurde: Wer Nahrungsmittel stahl und damit andere der Gefahr des Verhungerns aussetzte, sollte selbst dem Hungertod anheimfallen, siehe dazu v. Hentig (Anm. 1), S. 408; siehe auch die Beispiele bei Foucault (Anm. 9), S. 60.

159 Jakobs (Anm. 7), 1. Abschnitt Rdn. 10.

160 Vgl. Hegel (Anm. 96), \$ 99 (Z); dazu Mohr (Anm. 139), S. 95, 110.

161 Vgl. zur Abgrenzung von Recht und Moral nach den Reaktionen der Betrachter Ehrlich, Grundlegung der Rechtssoziologie, 1967, S. $131 \mathrm{ff}$. 
von Ehrenstrafen von den Wert- und Gefühlsdispositionen der Betrachter abhängig ${ }^{162}$. Dies betrifft einesteils die Wertbeziehung der Strafe zur Norm: In dem Maße, in dem bei der einen Gruppe von Betrachtern Schulterzucken, bei anderen aber Empörung hervorgerufen wird, verschwimmt die Berechenbarkeit der Übelszufügung. Weit schlimmer ist freilich, dass die Ehrenstrafen auch gegen die der Strafe als Rechtsinstitut immanente Wertbindung verstoßen, insofern sie die Betrachter als Resonanzboden zur Erreichung der Strafzwecke funktionalisieren. Was von den dabei angesprochenen und ausgelösten menschlichen Gefühlen zu halten ist, hat Kant in seiner Tugendlehre zum Ausdruck gebracht: Die Schadenfreude sei ein „geheime[r] Menschenhaß und das gerade Widerspiel der Nächstenliebe" und stehe in einem direkten Zusammenhang mit der Rache, die sich „den Schein des größten Rechts, ja wohl gar die Verbindlichkeit (als Rechtsbegierde)“ gebe ${ }^{163}$. Gerade wegen dieser Nähe lässlicher menschlicher Reaktionen und staatlicher Strafe besteht Kant auf einer strikten Trennung ${ }^{164}$. An anderer Stelle wird der dahinter stehende Gedanke ausformuliert: Nicht der homo phaenomenon als des Verbrechens fähiger Mensch fasse das Strafgesetz notfalls auch gegen sich selbst ab, sondern der homo noumenon. Demzufolge strafe auch nicht das Volk, also jede einzelne oder bestimmte einzelne Personen mit ihren individuellen, auch schlechten, Charakterdispositionen, sondern „das Gericht (die öffentliche Gerechtigkeit)“ 165 - wenn man so will: der institutionalisierte bomo noumenon. Indem gerade diese Instanz angerufen wird, erfüllt sich die „Forderung einer vom subjektiven Interesse und Gestalt sowie von

162 Pawlik (Anm. 12).

163 Kant (Anm. 68), S. 352, wonach Schadenfreude das „Wohlsein“ sei, „wenn Unglück oder Verfall anderer in Skandale gleichsam als Folie unserem eigenen Wohlstande untergelegt wird, um diesen in ein desto helleres Licht zu stellen“.

164 Kant (Anm. 68), S. 352: „Von dieser Schadenfreude ist die süßeste und noch dazu mit dem Schein des größten Rechts, ja wohl gar der Verbindlichkeit (als Rechtsbegierde), den Schaden anderer auch ohne eigenen Vorteil sich zum Zweck zu machen, die Rachbegierde. Eine jede das Recht des Menschen kränkende Tat verdient Strafe, wodurch das Verbrechen an dem Täter gerächt (nicht bloß der Schaden ersetzt) wird. Nun ist aber Strafe nicht ein Akt der Privatautorität des Beleidigten, sondern eines von ihm unterschiedenen Gerichtshofes, der den Gesetzen eines Oberen über alle, die demselben unterworfen sind, Effekt gibt, und wenn wir die Menschen (wie es in der Ethik notwendig) ist, in einem rechtlichen Zustande, aber nach bloßen Vernunftgesetzen, (nicht nach bürgerlichen) betrachten, so hat niemand die Befugnis, Strafen zu verhängen, als der, welcher auch der oberste moralische Gesetzgeber ist, und dieser allein (nämlich Gott), kann sagen: ,Die Rache ist mein; ich will vergelten“ “. Hingegen misst Durkheim (Anm. 88), S. 128 f., der Rache insoweit einen Wert bei, als sie eine - allerdings grobe Waffe zur Verteidigung vor Gefahren sei.

165 Kant (Anm. 68), S. 197. 
der Zufälligkeit der Macht befreiten, so nicht rächenden, sondern strafenden Gerechtigkeit“166. Die Ehrenstrafen rauben dieser Instanz das Exklusivrecht des Strafens, indem sie die Zeremonie der Strafe für das Publikum öffnen, es an der Vollstreckung der Strafe beteiligen, ja die Öffentlichkeit zum Vollstrecker der Strafe machen ${ }^{167}$. Damit aber fallen Strafanspruch und Strafvollstreckung partiell auseinander, steht doch die Berechtigung zur $\mathrm{Zu}-$ fügung eines dem Schaden am Recht äquivalenten Schmerzes allein der Rechtsgemeinschaft als "Gläubiger“ $\mathrm{zu}^{168}$. Diese Gläubigerstellung ist konsequente Folge der Verletzung ihrer Rechts- und Friedensordnung, mit welcher wir oben die grundsätzliche Relevanz ihrer Straferwartung normativ begründet haben. Als Rechtsgemeinschaft ist freilich nicht die Masse empirischer Menschen mit ihren individuellen Dispositionen zu verstehen, sondern die Gemeinschaft von Bürgern, welche die Durchsetzung des Rechts an Verfahren und Institutionen delegiert hat. Strafen darf im System der Rechtsstrafe also nicht irgendeine Gruppe von Individuen mit ihren zufälligen Dispositionen. Indem shame sanctions ihre Existenz wesentlich den Reaktionen eben solcher Personen verdanken, wird diese tragende Säule der Rechtsstrafe unterminiert. Der in der Ehrenstrafe liegende Systembruch wird noch deutlicher, wenn man die Perspektive wechselt: Der Delinquent wird zur Duldung der Strafvollstreckung durch eine Personengruppe verpflichtet, die nicht Inhaber des Strafanspruchs ist und der gegenüber er folglich auch nicht zur Duldung der Strafvollstreckung verpflichtet ist. Dieser Systembruch ist nicht auf das Strafrecht beschränkt, sondern tangiert das grundsätzliche Verhältnis der Staatsbürger in einer Rechtsgemeinschaft: Kant hat in seiner Staatslehre unmissverständlich als „Attribut der bürgerlichen Selbständigkeit“ eingefordert, dass jeder in seiner „Existenz und Erhaltung

166 Hegel (Anm. 96), \$103. Durkheim (Anm. 88), S. 137 f., erachtet diese organisatorische Verlagerung bei der Ermittlung und der Durchführung der Strafe an eine von den Einzelnen zu unterscheidende Körperschaft sogar als den Wesenszug der Strafe, welcher sich als das dauerhafte Element in allen gesellschaftlichen Formen des Strafens erwiesen habe.

167 So Schwerhoff (Anm. 26), S. 159; Schild, Alte Gerichtsbarkeit, 1985, S. 212; siehe auch Whitman (Anm. 2), S. 1059: „The chief evil in public humiliation sanctions is that they involve an ugly, and politically dangerous, complicity between the state and the crowd".

168 Vgl. Nietzsche (Anm.120), S. 292f., 302. Eine soziologische Erklärung liefert Durkheim (Anm. 88), S. 144 f.: Indem das Verbrechen jeden Einzelnen berührt und in allen gleichgerichtete Gefühle auslöst, schließen sich die Einzelnen in ihrer Reaktion als Gemeinschaft enger zusammen; die „Masse verdichtet“ sich, vgl. Canetti (Anm. 32), S. $23 \mathrm{ff}$. 
nicht der Willkür eines anderen im Volke“ ausgeliefert wird ${ }^{169}$. Auch der Delinquent darf also nicht der Reaktion irgendwelcher Personen ausgesetzt werden, sondern allein der strafenden Macht des Staates, dessen Mitträger er ist.

Erst an dieser Stelle sieht sich also das intuitive Unbehagen gegenüber den shame sanctions normativ bestätigt. Wer Ehrenstrafen propagiert, muss diesen Systembruch eingestehen.

\section{Zusammenfassung}

Die Untersuchung hat gezeigt, dass die shame sanctions ihrer drastischen Symbolik zum Trotz keineswegs Ausdruck einer plakativen Rechtspolitik sind, sondern sich in die kriminalpolitische Entwicklung der USA einpassen lassen. Beunruhigend für den deutschen Beobachter ist der Befund, dass Ehrenstrafen grundsätzlich mit den wesentlichen Sinnzuschreibungen an die Strafe vereinbar sind. Die in der amerikanischen Diskussion vorgebrachten Gegenargumente haben sich als nicht stichhaltig erwiesen. Hilfreicher sind demgegenüber die von Kant und namentlich Hegel ausgearbeiteten strafrechtstheoretischen Grundlagen. Es mag zwar ungerecht erscheinen, eine Strafform, die das Gepräge des Mittelalters hat, an den Erkenntnissen des Idealismus zu messen. Soll die Kriminalpolitik aber nicht hinter diese Einsichten zurückfallen, muss sich ihre Ausgestaltung der Sanktionen vor den strafrechtstheoretischen Lehren dieser Zeit behaupten können. Die Strafe als Wiederherstellung des Rechts als Recht darf sich zwar einer tadelnden Übelszufügung bedienen. Versteht man die Strafe aber als wertbezogene und wertgebundene Institution werden innersystematischen Vorgaben sichtbar, an welchen die shame sanctions gemessen werden können. Die Wertbindung einer Strafe, welche die Geltung des Rechts manifestiert, verlangt eine Förmlichkeit des Verfahrens zumindest insoweit, als die Vollstreckung nicht Individuen mit ihren unterschiedlichen und zufälligen charakterlichen Dispositionen überantwortet werden darf: Der Strafanspruch steht der Rechtsgemeinschaft zu. Ihr und den von ihnen vorgesehenen Institutionen ist der Delinquent zur Duldung der Strafvollstreckung verpflichtet - nicht einer zufälligen Masse von Passanten.

169 Kant (Anm. 68), S. 171. 
Gut und Böse wohnen in jedem Individuum dicht beieinander. Den Menschen kann man, wie Nietzsche daher meinte, mit der Strafe nicht bessern, sondern allenfalls zähmen ${ }^{170}$. Gerade dann aber darf die Zähmung nicht von jenen vorgenommen werden, die sich konstitutionell selbst nicht immer im Zaume halten können.

170 Nietzsche (Anm. 120), S. 317. 